\title{
Nobeyama radio heliograph observations of RHESSI microflares
}

\author{
M. R. Kundu ${ }^{1}$, E. J. Schmahl ${ }^{1,2}$, P. C. Grigis ${ }^{3}$, V. I. Garaimov ${ }^{4}$, and K. Shibasaki ${ }^{5}$ \\ 1 Astronomy Dept., University of Maryland, College Park, MD 20742, USA \\ e-mail: ed@astro.umd.edu \\ 2 Lab for Solar and Space Physics, NASA/GSFC, Code 612.1, Greenbelt, MD 20771, USA \\ ${ }^{3}$ Institute of Astronomy, ETH Zurich, Switzerland \\ ${ }^{4}$ Special Astrophysical Observatory, St. Petersburg, Russia \\ 5 Nobeyama Radio Observatory, Minamimaki, Nagano, Japan
}

Received 4 August 2005 / Accepted 19 January 2005

\begin{abstract}
Aims. We present a summary of the analysis of thirty microflares, observed simultaneously by the Ramaty High Energy Solar Spectroscopic Imager (RHESSI) in hard X-rays and by Nobeyama RadioHeliograph (NoRH) in microwaves (17 GHz).

Methods. We used microflares observed by RHESSI in the energy range 3-25 keV, and for larger events, up to $35 \mathrm{keV}$. The observations were made 2002, May 2-6.

Results. We describe the imaging characteristics of these microflares including their locations in hard X-rays and microwaves and the relative positions of the micro-flaring sources. We discuss the brightness temperatures, emission measures and their hard X-ray spectral properties. We see small (mini) flaring loops clearly in NoRH and RHESSI images. The microwave emission often seems to come from the RHESSI foot points (for higher energies), and from the entire small (mini) flaring loop (for lower energies). Sometimes the two (microwave and hard X-ray) sources coincide, at other times they are at opposite ends of a mini flaring loop. Typically, the hard X-ray spectrum of the microwave associated RHESSI microflares can be fit by an isothermal component at low energies (below 10 or $12 \mathrm{keV}$ ) and a nonthermal component at higher energies (above $12 \mathrm{keV}$ ).

Conclusions. Microflares in hard X-rays and in microwaves behave like normal flares in many respects. They can have both thermal and nonthermal components appearing in bremsstrahlung and gyrosynchrotron radiation.
\end{abstract}

Key words. flares - Sun: photosphere - Sun: X-rays, gamma rays - Sun: corona

\section{Introduction}

The Sun releases energy in transient bursts ranging from major flares $\left(10^{32}-10^{33} \mathrm{erg}\right)$ down to microflares and nanoflares. The occurrence frequency of these transient releases increases with decreasing energy. For flares, the energy releases are dominated by several 10's to several 100's of keV electron energy; and for hard X-ray microflares with energies of $10^{26}-10^{27} \mathrm{erg}$, they are dominated by electrons with energy $<20 \mathrm{keV}$ to $8 \mathrm{keV}$ (Lin et al. 1984; Lin et al. 2001).

Observations made over the past several years with the soft and hard X-ray telescopes (SXT and HXT) on the Japanese satellite Yohkoh have resulted in many new and important discoveries. For example, they have revealed that the X-ray corona is much more dynamic than had been suspected before. Because of the high spatial resolution and the large dynamic range of SXT, it has been possible to discover varieties of small scale energy releases on the Sun and to study them with extraordinary sensitivity and detail. As a result, we now know of several new phenomena: (a) XBP flares and their extended structures; (b) coronal X-ray jets; (c) Active Region
Transient Brightenings (ARTB's); and (d) X-ray plasmoid ejections. Similarly, the SOHO/EIT/SUMER/UVCS experiments have revealed many transients such as eruptive plumes, macro spicules, etc. in EUV images. All these small scale phenomena have their distinctive morphological and physical properties in $\mathrm{X}$-rays, and their interpretations, although related, can be quite different.

In this paper we present the results of a study of microflare phenomena focusing on additional information provided by radio data. Radio observations provide information complementary to that of soft X-ray or EUV images: the latter two are both dominated by thermal emission from coronal plasma, whereas radio observations are also sensitive to nonthermal emission and to much cooler plasma. However, radio observations are not always the most efficient way to search for transients because radio observed morphological structures of weak transients take rather simple forms. Hence most radio studies of transients have used X-ray selected events.

In the radio domain, small-scale energy releases are essentially weak coronal transients. The observational characteristics 
of X-ray bright point flares at meter wavelengths and in microwaves show that both thermal and nonthermal processes occur in these small-scale flaring events. Similarly, radio observations of X-ray jets in microwaves and at meter wavelengths provide evidence for both thermal and nonthermal processes in these dynamic coronal phenomena. Nonthermal radio emission in the form of metric type III bursts is produced by electron beams propagating along the jet, whereas microwave emission mostly comes from the jet base. The radio emission from active region transient brightenings (ARTB's) can be purely thermal, thermal gyro-resonance or nonthermal gyrosynchrotron radiation. The quiet Sun transient brightenings located far from active regions and those occurring on network boundaries (network flares) as well as weak plasmoid ejections can also be thermal or nonthermal. These transients are at least two orders of magnitude weaker than normal flares both in X-ray and radio domains. Thus, one would like to think of them as microflares with different morphological manifestations in the X-ray domain. In the radio domain, one sees distinct signatures of nonthermal and thermal processes in them. In the case of radio thermal emission, one sometimes observes more emission than that computed from the plasma parameters of the thermal X-ray plasma; this may simply imply that the cooler material is seen in radio, but not in X-rays. The findings on the radio counterparts of X-ray transients have been reviewed by Kundu (1998).

The Reuven Ramaty High-Energy Solar Spectroscopic Imager (RHESSI) instrument (see Lin et al. 2002) provides high sensitivity in the energy range from $\sim 3$ to $15 \mathrm{keV}$, together with $\sim 1 \mathrm{keV}$ FWHM spectral resolution and imaging with 2.3 arcsec resolution. This energy range covers emissions from hot $(\sim 5 \mathrm{MK})$ thermal plasmas and from accelerated, nonthermal low-energy electrons. Previous solar hard X-ray instruments, such as HXRBS flown on SMM and HXT on Yohkoh had entrance windows that absorb emission below $15-25 \mathrm{keV}$ to avoid saturation from the intense thermal emissions in large flares. The RHESSI instrument accommodates medium and large flares by automatically inserting shutters in front of detectors to absorb low-energy photons and avoid saturation. With shutters out, RHESSI's front-segment effective area (not including the transmission of the grids) is $\sim 1 \mathrm{~cm}^{2}$ at $4 \mathrm{keV}$, rising rapidly to $\sim 60 \mathrm{~cm}^{2}$ at $6 \mathrm{keV}$, and plateauing near $\sim 200 \mathrm{~cm}^{2}$ in the 10-100 keV range. (Smith et al. 2002) The effect of the grids is to reduce these areas by about a factor of 4 . This is about a factor of 14 to $\sim 130$ times larger than the comparable instrument HXIS on SMM. (Van Beek 1980) Thus, the RHESSI observations from 3 to $15 \mathrm{keV}$ with the shutters out can provide new information on low-level energy releases, whether they result in heating of hot thermal plasmas or in the acceleration of low-energy electrons.

The first observations of microflares in the energy range $3-15 \mathrm{keV}$ were made by Krucker et al. (2002) and Benz \& Grigis (2002) with open-shutter RHESSI. Even during periods of lowest solar activity, ARTB's and microflares were detected at a rate of at least 10 per hour. A thermal component fitted by temperatures of 6-14 MK dominates from $3 \mathrm{keV}$ to about $9 \mathrm{keV}$, but can be observed up to $14 \mathrm{keV}$ in some cases, and has an average duration of $131 \pm 100 \mathrm{~s}$ at $7-8 \mathrm{keV}$. The duration seems to increase with decreasing photon energy.
The temperature peaks early in the event and then decreases, whereas the emission measure increases throughout the event. In some of the larger events, a second component was detected in the $11-14 \mathrm{keV}$ range going down to $8 \mathrm{keV}$ in some cases. Its duration is typically 3 times shorter and it ends near the peak time of the thermal component, consistent with the Neupert effect of regular flares. Thus the second component is inferred to be of nonthermal origin.

In this paper, we plan to compare hard X-ray and radio data for microflares using RHESSI in conjunction with NoRH at $17 \mathrm{GHz}$. Hard X-rays (>20 keV) are very closely associated with microwave emission from normal flares and frequently they are associated with type III bursts. We make a detailed study of 3-25 keV microflares as observed by RHESSI with simultaneous imaging observations in microwaves by NoRH. The objective is to understand the nature of microflares better and in particular using microwave and hard $\mathrm{X}$-ray data that are ideally suited for this purpose, to investigate the nonthermal aspects of microflares that are not addressed by EUV or soft X-ray data that have been the tools for most studies of these events.

\section{Observing procedure}

RHESSI was launched in Feb. 2002 to observe solar flares in the energy range $3 \mathrm{keV}$ to $17 \mathrm{MeV}$, with energy resolution of $\sim 1 \mathrm{keV}$ at low energies. It is the most powerful space mission to observe solar flare emission produced by nonthermal electrons. It can operate with and without shutters, and therefore it is able to detect very weak events such as microflares. For our study we used RHESSI-selected events observed in the shutter-free mode. Standard software is used to retrieve RHESSI light curves of selected events at a few energy ranges in the $3-25 \mathrm{keV}$ range, which we compare with microwave imaging observations made with NoRH at 17 and $34 \mathrm{GHz}$. At these two frequencies NoRH has a spatial resolution of $\sim 10^{\prime \prime}$ and $5^{\prime \prime}$ respectively. We also used NoRP polarimeter data (full-sun monitoring data) at 1.0, 2.0, 3.75, 9.4, 17 and $34 \mathrm{GHz}$ - whenever available. NoRH provides both $I, V$ Stokes parameters at $17 \mathrm{GHz}$ and only $I$ at $34 \mathrm{GHz}$. NoRP provides $I, V$ at most frequencies. RHESSI spatial resolution is typically $\sim 7^{\prime \prime}$ using subcollimators $3-9$, but can be as good as 2.3" if subcollimators 1 and 2 can be used. With the shutters out, RHESSI can detect individual photons down to $3 \mathrm{keV}$, and can make rough images with as few as $\sim 10^{3}$ to $10^{4}$ photons at higher energies. NoRH has a sensitivity of at least $1 \mathrm{sfu}$ at $17 \mathrm{GHz}$ and $\sim 3 \mathrm{sfu}$ at $34 \mathrm{GHz}$. The normal time resolution is $1 \mathrm{~s}$, but a resolution as good as $50 \mathrm{~ms}$ can be used for special projects. For our study we've used $1 \mathrm{~s}$ time resolution.

\subsection{Microwave and Hard $X$-ray imaging observations: general overview}

As mentioned earlier we've studied microwave counterparts of RHESSI-selected microevents. Thirty microwave events corresponding to RHESSI microflares were selected in the Nobeyama time zone for our study during the period 


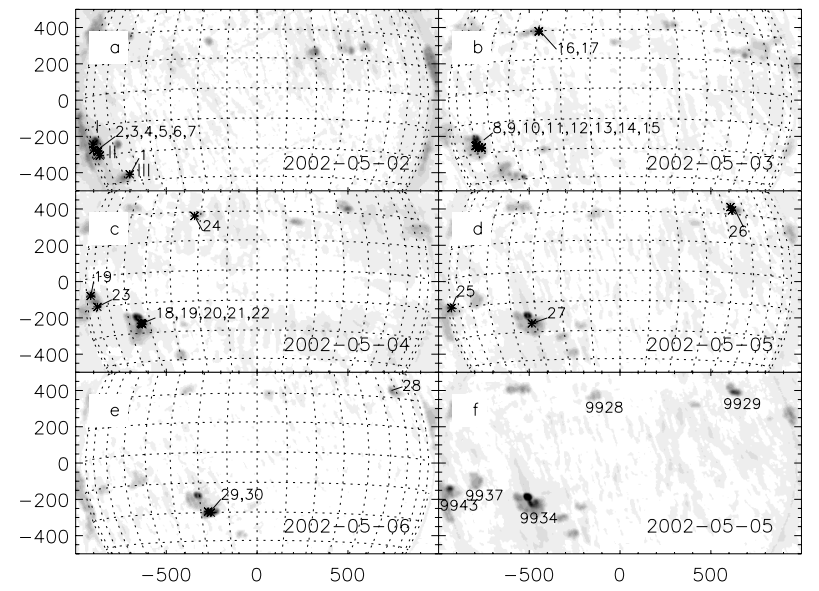

Fig. 1. A piece of the Sun for each day (2002 May 2-6), showing the locations of the microflares in this study. The panel f) shows the white light (MDI) image for 2002 May 5.

2002 May 2-6. Several active regions including AR 9934 were involved in the microflaring process. We shall discuss only some events observed in the Nobeyama time zone during this period. AR 9934 was a complex region which contained a sunspot with a strong negative polarity of the magnetic field on the north side and a bipolar region on the south side. MDI images show fast evolution of the south part of the region. TRACE images show many small loops in the south and a complex loop connecting the north sunspot to the south side of the region.

Figure 1 shows a piece of the Sun for each day (May 2-6, 2002), containing the regions in which the microflares occurred. Figure 2 shows for a typical microflare (01:52 UT event of May 2) 17-GHz and RHESSI light curves, the corresponding RHESSI spectrogram, and RHESSI and $17 \mathrm{GHz}$ image contours superposed on the MDI magnetogram and the EIT image. As mentioned earlier, the MDI magnetogram shows evolution in the southern part of the active region, and both HXR and microwave sources seem to be located in projection over a small EIT loop. Figures $3 \mathrm{a}-\mathrm{i}$ show $17-\mathrm{GHz}$ NoRH and RHESSI 3-6 keV light curves from the various flaring regions. The flux profiles are constructed from maps whose background brightness levels are scaled to $10^{4} \mathrm{~K}$. We have estimated the relative noise level from the flux of subregions of low $T b$; these show fluctuations of $0.01-0.02 \mathrm{~K}$ on timescales of minutes. This noise level is indicated in the captions of each profile. Figures $4 \mathrm{a}-\mathrm{k}$ show the RHESSI microflare images (contours) superposed on the $17-\mathrm{GHz}$ images. Note that for each microevent there are 3 RHESSI images (except when the higher energy bands have insufficient counts for mapping) in the energy ranges 3-6 keV, 6-12 keV and 12-25 keV.

NoRH 17-GHz maps of the second microburst on May 2 (Fig. 2) show a maximum above the sunspot in the north; there is also emission on the south side of the AR. RHESSI maps superposed on the NoRH maps show that X-ray emission in the range $3-25 \mathrm{keV}$ is located inside the radio contours. During most of the events on this day (\#2, 3, 5, 6 and 7, Figs. $4 a-c)$ ), HXR emission is located in the south part of the AR. RHESSI maps for 2002 May 3, events \#8-12, 14 and 15 , clearly show an X-ray loop at 3-6 keV and in events \#11 and 12, at least one footpoint of the loop may be seen in the 6-12 and 12-25 keV ranges. These footpoints are located above opposite magnetic polarities as seen in overlays of hard X-ray images on the MDI image.

For 2002 May 4 HXR images (Fig. 4h, events \#18-22 and Fig. 4g, event \#23) show a small X-ray feature occurring at nearly the same position about $30^{\prime \prime}$ away from the $17-\mathrm{GHz}$ source. No footpoints of a putative X-ray loop are seen. Overlays of HXR images on the MDI magnetograms show that the X-ray source is sometimes located above the magnetic neutral line connecting two regions with opposite magnetic polarities. This suggests that we are dealing with small (mini) loops. In all events HXR emission was observed in the 6-12 keV band and sometimes in the $12-25 \mathrm{keV}$ band. Total radio flux from the $\mathrm{X}$-ray emitting active region was less than $0.5 \mathrm{sfu}$. Significant polarization of the radio emission was observed in many cases. (See Figs. 2 and 9.)

\section{Specific examples}

Figures $3 \mathrm{a}-\mathrm{i}$ show the time profiles of NoRH 17-GHz flux for 2002 May 2-6. The dotted vertical lines in each panel show the times of NoRH maps and the solid dark lines at the bottom of each panel show the time intervals during which the RHESSI maps were made.

The 2002 May 2, 01:52 event is one of several microflares that originated in the NE part of the active region. At 01:52:10 UT RHESSI sources in 3 energy bands overlie a 17-GHz SW source which occupies mostly an MDI negative polarity (see Fig. 2). The NE source and another SW source seem to contribute to the microflare emission at the same time as judged from the time profiles. Several components of the region concerned seem to be responsible for different microflares on the same day.

\subsection{May 02 microevents}

On this day we observed 7 microflares, \#1-7 (see Fig. 3a). Figures $4 a-c$ show the 7 microevents observed at 01:47, 01:52, 02:00, 02:10, 02:21, 02:30 and 02:37 UT. The figures show RHESSI contour maps superposed on NoRH $17-\mathrm{GHz}$ maps. The 17-GHz maps show two sources of which the northern one must be a sunspot-associated gyro-resonance source. The HXR source in 3-6, 6-12 and 12-25 keV bands agrees quite well with the $17-\mathrm{GHz}$ source, although there is a small limbward displacement of the HXR source relative to the $17-\mathrm{GHz}$ sources. The same situation holds for microevents at 02:10, 02:21, 02:30 and 02:37 UT. At 02:10 UT the HXR source is closer to the sunspot source and at 02:21 UT it is closer to the 17-GHz transient source in the south and it seems to have elongated structures. Note that in the background-subtracted images at 01:52 the HXR source in the $12-25 \mathrm{keV}$ band coincides with the $17-\mathrm{GHz}$ source. The only time that the HXR source is strong enough to be mappable in this energy range is for the events \#1 at 01:47 and \#2 at 01:52. 

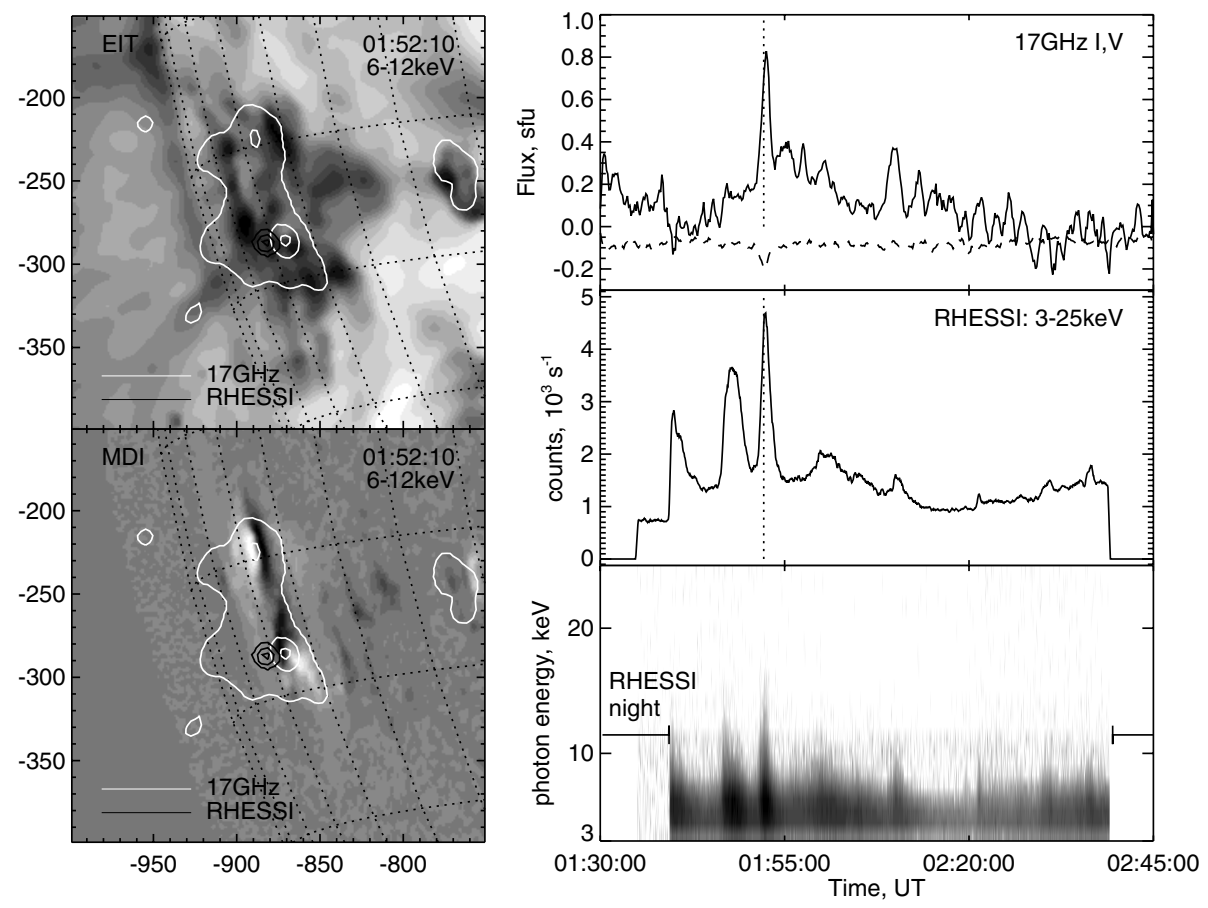

Fig. 2. Microflare event of 2002 May 2, 01:52 UT. 17-GHz and RHESSI light curves, the corresponding RHESSI spectrogram, and RHESSI and $17 \mathrm{GHz}$ image contours superposed on the MDI magnetogram and the EIT image.

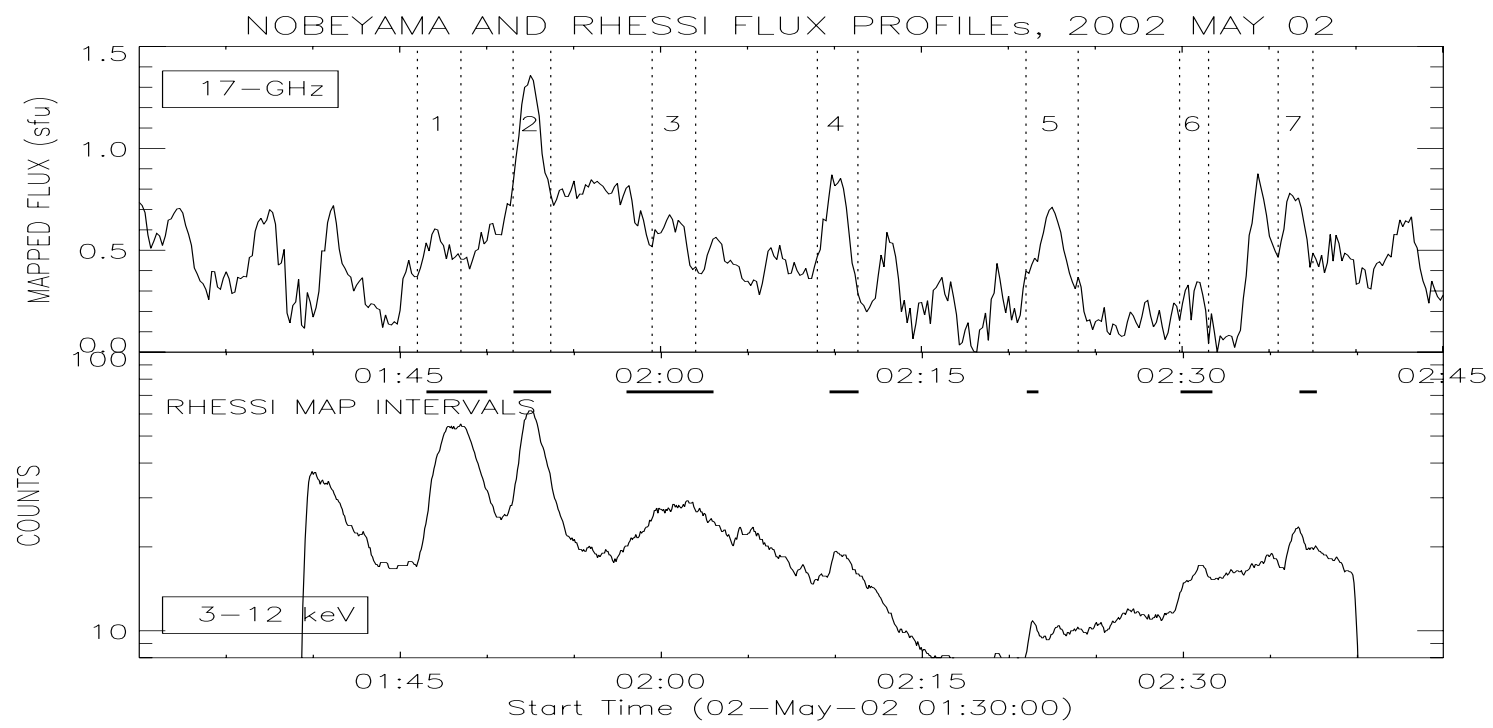

Fig. 3a. 17-GHz NoRH and RHESSI 3-6 keV light curves for events 1-7. The vertical dashed lines show the intervals used for 17-GHz mapping. Similarly, the horizontal bars show the intervals used for HXR mapping. Based on samples of background submaps, the noise in the time profile is $0.01 \mathrm{~K}$.

\subsection{May 03 microevents}

We show in Figs. 4d-g images of ten microevents in HXR and $17 \mathrm{GHz}$ at 03:28, 03:45, 03:49 (events \#8-10 in Fig. 4d), 03:55, 03:58, 04:15 (events \#11, 12 and 14 in Fig. 4e), 04:01, 05:06, and 05:09 (events \#13, 16 and 17 in Fig. 4f), and 04:16 UT (event \#15 in Fig. 4g).

The HXR microflares in Figs. 4d and 4e are single sources, displaced slightly south from the $17-\mathrm{GHz}$ sources, and both HXR and 17-GHz sources have loop-like elongated structures. Each HXR source has significant emission in the $12-25 \mathrm{keV}$ band. Note that the loop-like structures are more prominent for events \#8, 9, 14 and 15. The 03:55 microflare difference map for event \#11 (bottom row of Fig. 4e) shows a loop-like feature connecting the HXR source to the bright, compact $17-\mathrm{GHz}$ component $\sim 100^{\prime \prime}$ to the north. This is what one would expect from a normal flare in an asymmetric magnetic field.

The 03:58 microflare (\#12, Fig. 4e) is homologous to the earlier one in the two lower HXR bands as well as in microwaves. But in the $12-25 \mathrm{keV}$ band there is a secondary compact source located at what might be considered the apex of the 03:55 (\#11) loop. Note also the foot points of the HXR loop which are most prominent in the $12-25 \mathrm{keV}$ band. 


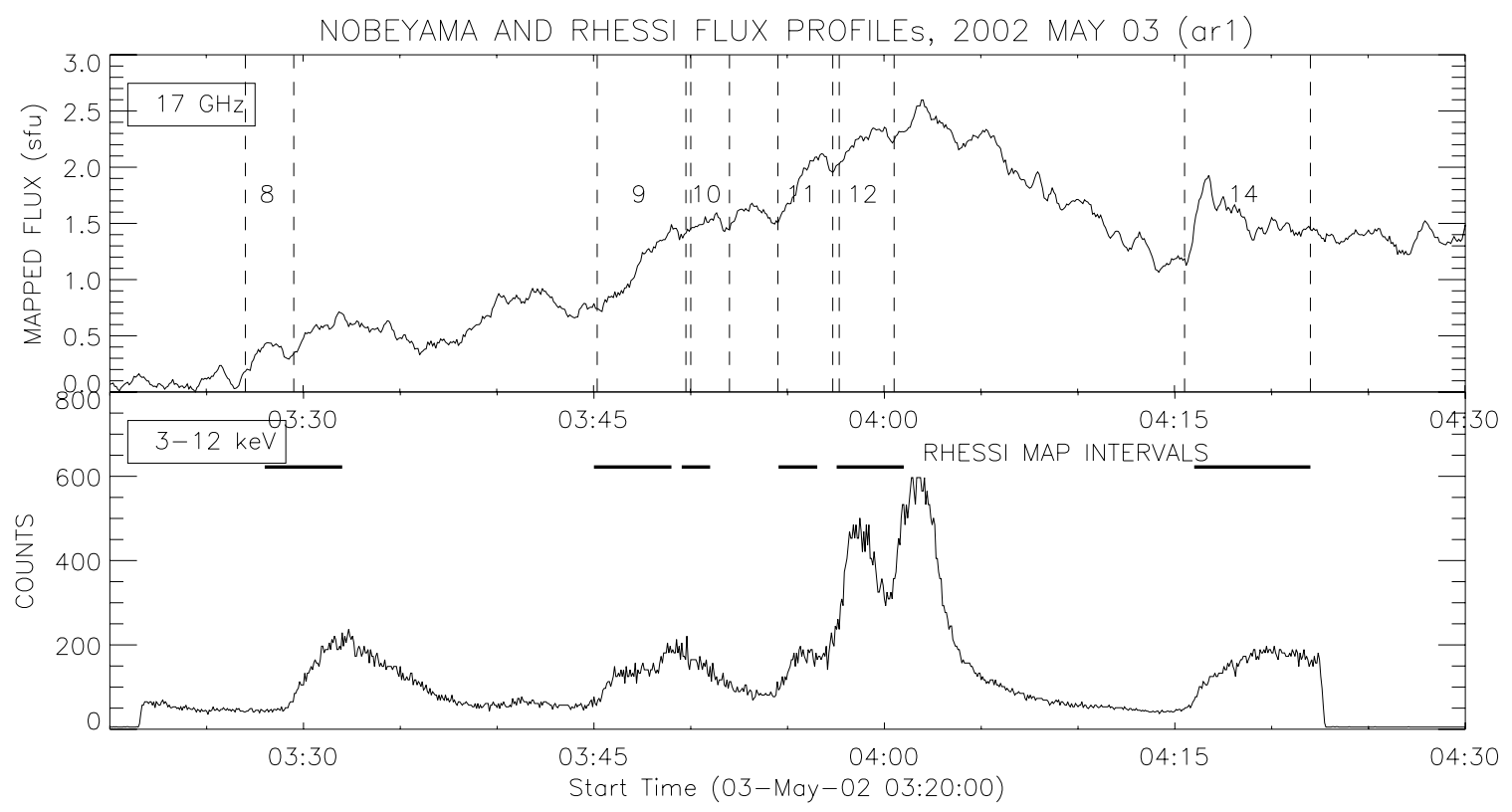

Fig. 3b. 17-GHz NoRH and RHESSI 3-6 keV light curves for events 8-12 and 14. The noise level in the $17 \mathrm{GHz}$ profiles is $\sim 0.014 \mathrm{~K}$.

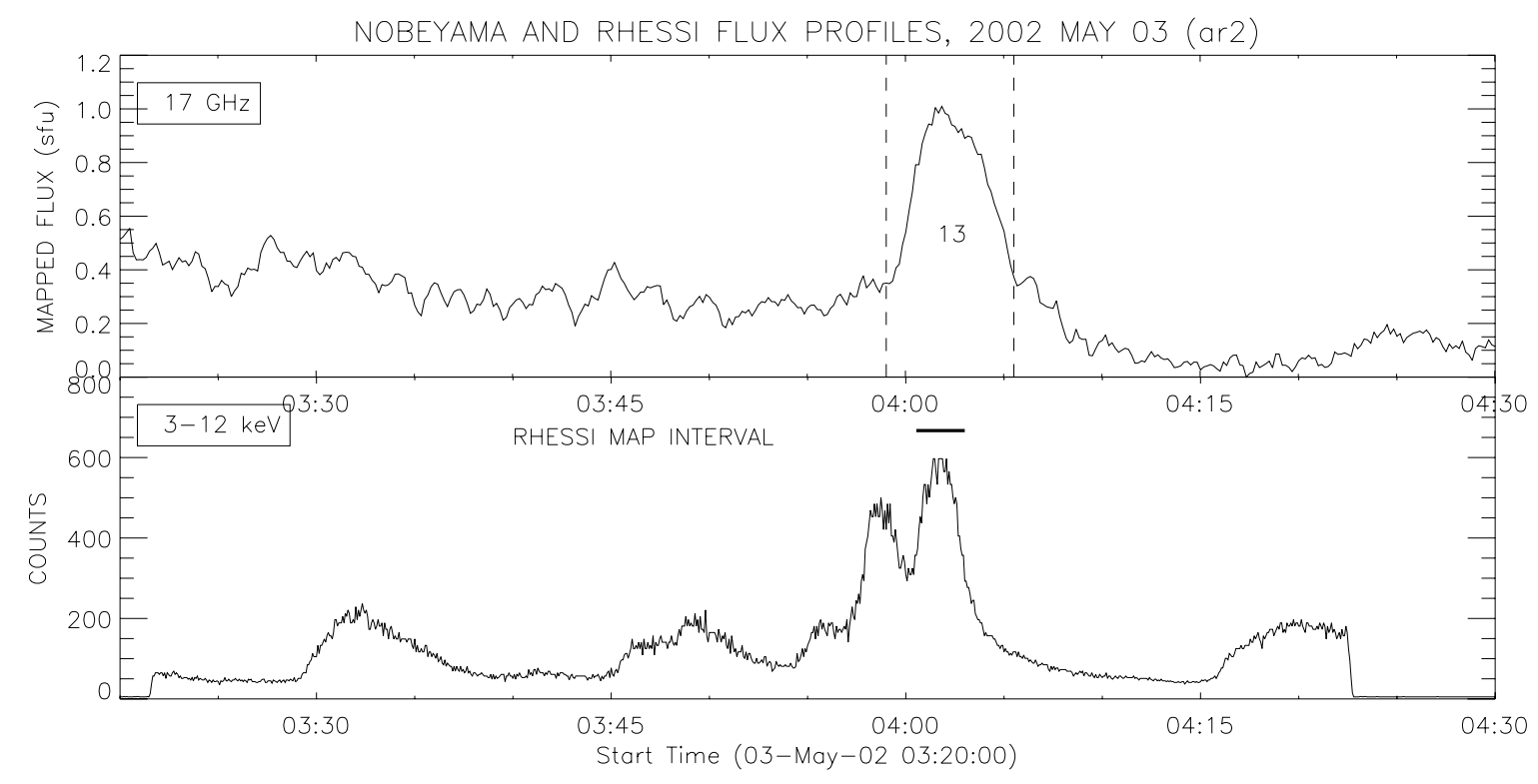

Fig. 3c. 17-GHz NoRH and RHESSI 3-6 keV light curves for event \#13. The noise level in the $17 \mathrm{GHz}$ profiles is $\sim 0.015 \mathrm{~K}$.

In the 04:15 event (\#14, Fig. 4e, 3rd column), the HXR source is single, but elongated, suggestive of a loop.

In Fig. 4f we show three microflares (\#s 13, 16 and 17) at 04:01, 05:06, and 05:09 UT. The first of these microflares (observed at 04:01) is very compact, and is co-spatial in HXR and $17 \mathrm{GHz}$. This event seems to have occurred in a relatively quiet region. The other two HXR microevents at 05:06 and 05:09 coincide rather well with the NoRH 17-GHz positions, with only a slight offset from the 17-GHz sources and have weak secondary components. Note that the background-subtracted $17-\mathrm{GHz}$ source is close to the 12-25 keV HXR source; the negative $17 \mathrm{GHz}$ minimum north of the HXR source appears to be an artifact resulting from the time variation of the background. For the 04:16 microevent (event $\# 15$, Fig. 4g), there is a displacement in position between the microwave and HXR flaring sources (HXR source being $\sim 30^{\prime \prime}$ to the south of the microwave source) in all three energy bands 3-6, 6-12, 12-25 keV, and the HXR source has a loop-like shape.

\subsection{May 04 microevents}

On this day we observed 7 microevents (\#s 18-24): 05:08, 05:14, 05:22, 05:38, 05:46, 05:54, and 05:59 UT. Figures $4 \mathrm{~h}-\mathrm{i}$ show events \# 18-22 and 24; Fig. 4g shows the 05:54 event \#23 (in a different region) in HXR and at $17 \mathrm{GHz}$. HXR emission is observed in the two lower energy channels, but not at the highest energy except for two events (\#18 at 05:08 and \#21 at $05: 38$ ). The HXR source is displaced from the $17-\mathrm{GHz}$ source; it is possible that the HXR and microwave sources are 


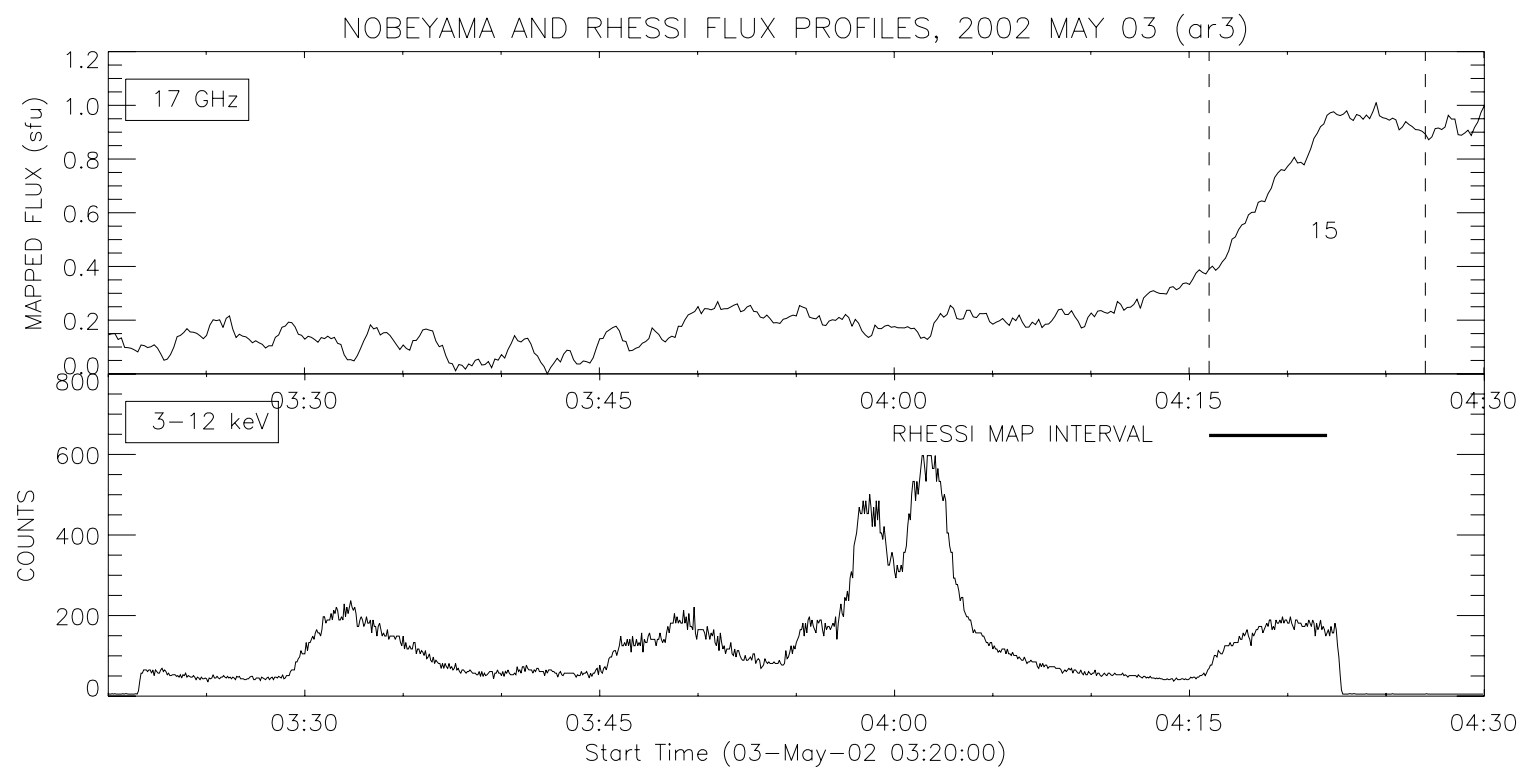

Fig. 3d. 17-GHz NoRH and RHESSI 3-6 keV light curves for event \#15. The noise level in the $17 \mathrm{GHz}$ profiles is $\sim 0.017 \mathrm{~K}$.

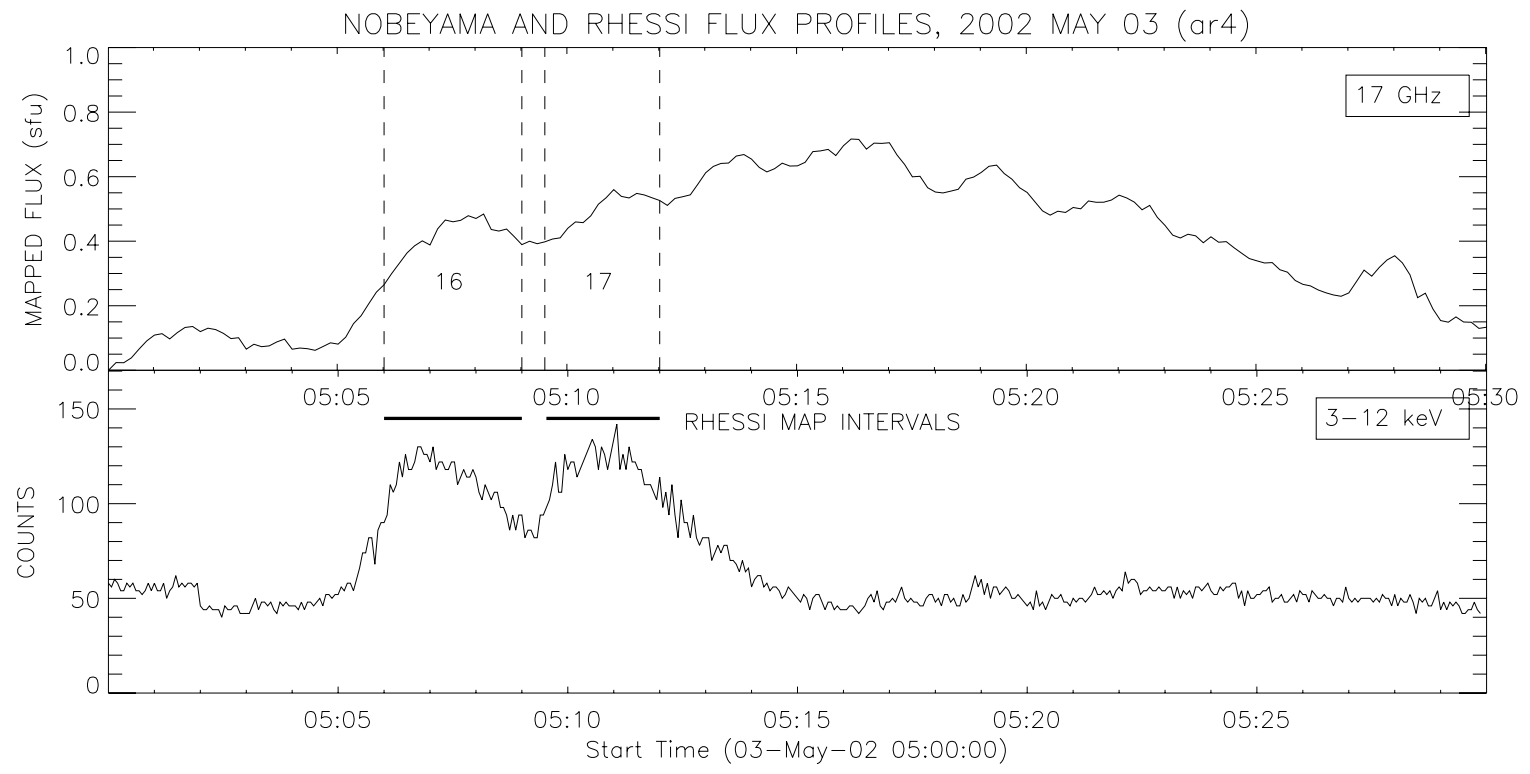

Fig. 3e. 17-GHz NoRH and RHESSI 3-6 keV light curves for events 16 and 17 . The noise level in the $17 \mathrm{GHz}$ profiles is $\sim 0.013 \mathrm{~K}$.

at the two ends of an unfilled loop. Note that the 17-GHz map for event \#24 at 05:59 UT is at a different location. This location and its field of view (FOV) is quite different from the locations and FOVs of the other 5 microflares. The 05:54 microflare (event \#23, Fig. 4g) comes from a region near the E-limb, and the HXR emitting loop agrees fairly well with the $17-\mathrm{GHz}$ region, located just to the south of it. There is a $17-\mathrm{GHz}$ source at the limb, which appears much brighter in the backgroundsubtracted map, and it is possible that the RHESSI source is at the other end of a loop connecting the limb 17-GHz source and the HXR source. Indeed, this loop is visible in the background subtracted map. There is no HXR emission in the higher energy 12-25 keV band.

An explanatory remark with regard to event \# 19 of May 4 is in order. Microevents \#18, 19 and 20 occurred near the strong, slowly-varying gyro-resonance source in this active region (Fig. 4h). All three microbursts occurred in a faint loop extending SW from the g-r source. Background subtraction is difficult in these maps because slow changes in the g-r source swamp the smaller microflare. Thus the backgroundsubtracted images (Fig. 4h) show negative "shoulders" bordering the strong g-r source. Event \#19 is peculiar in another respect. The HXR event is relatively weak (see the time profile in Fig. $3 f$ for these events), considering the strength of the $17-\mathrm{GHz}$ event compared to the other microflares on May 4. To complicate matters, coincidentally there was a near-simultaneous HXR microflare close to the limb (Fig. 1c), located 200" east of these 3 microflares. Because of the stronger and harder limb event (which, curiously, did not appear as a microflare in the 17-GHz full-disk maps, but only as a slowly-varying 
NOBEYAMA AND RHESSI FLUX PROFILES, 2002 MAY 04

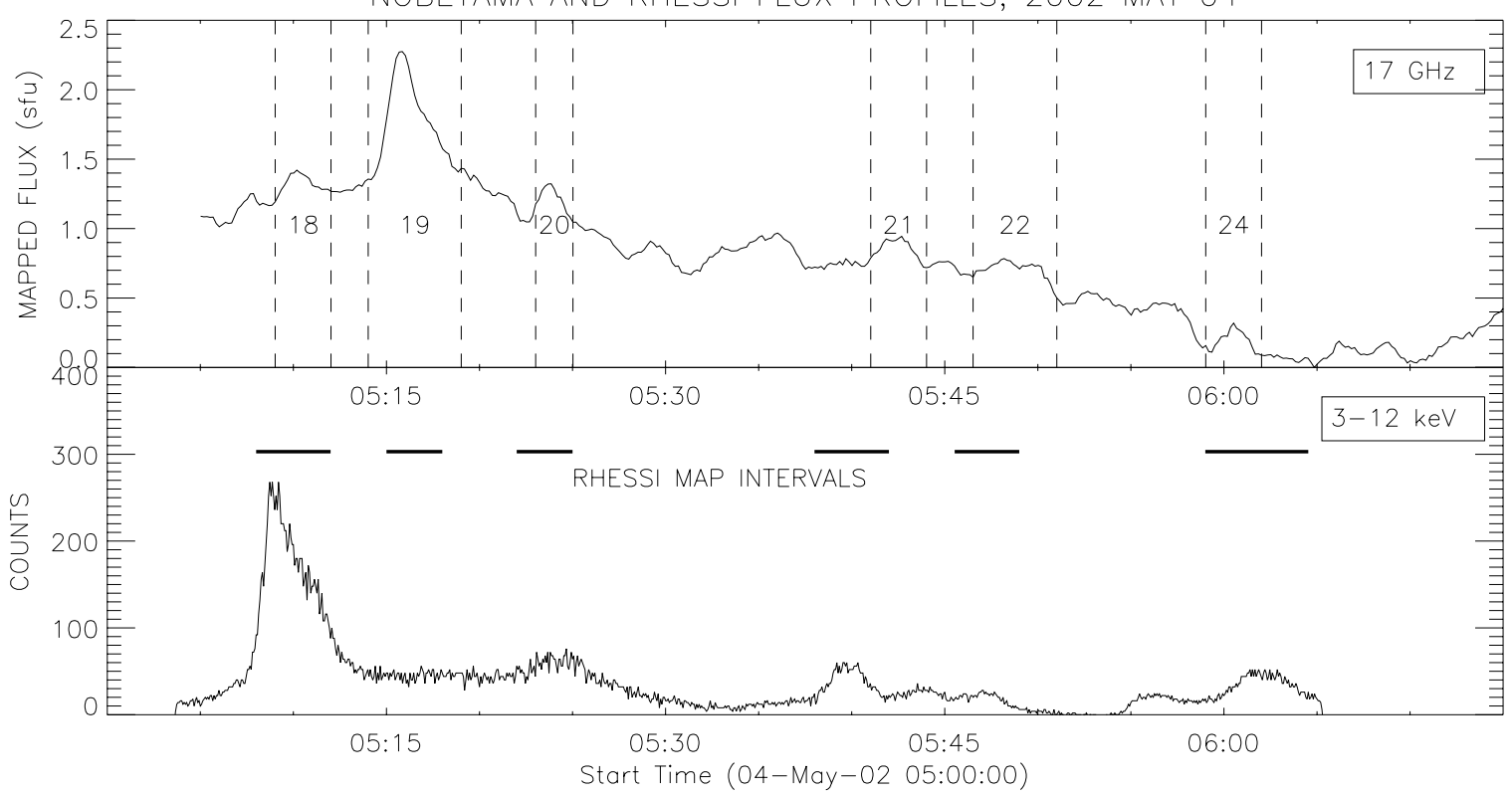

Fig. 3f. 17-GHz NoRH and RHESSI 3-6 keV light curves for events $18-22$ and 24 on May 4 . The noise level in the $17 \mathrm{GHz}$ profiles is $\sim 0.016 \mathrm{~K}$.

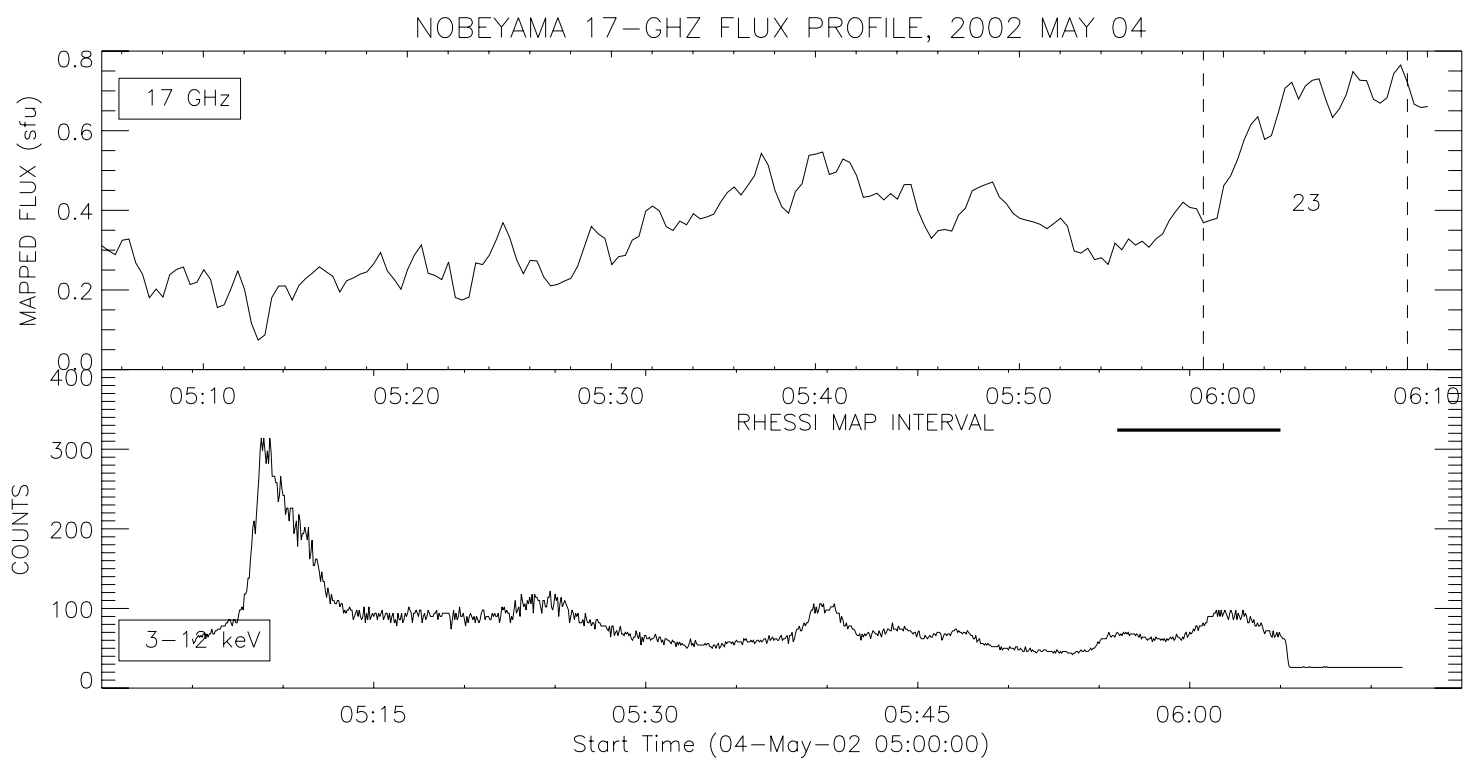

Fig. 3g. 17-GHz NoRH and RHESSI 3-6 keV light curves for event \#23. The noise level in the $17 \mathrm{GHz}$ profiles is $\sim 0.010 \mathrm{~K}$.

g-r source), RHESSI Clean maps had to be made with somewhat degraded spatial resolution ( $12^{\prime \prime}$ instead of $\left.7^{\prime \prime}\right)$. Thus the apparent increased size of the HXR source in event \#19 is only the result of lower spatial resolution.

\subsection{May 05 microevents}

On May 5, three microevents were observed in three different locations (events \#25-27): 03:46, 03:55, and 03:59 UT. For all 3 events, RHESSI HXR sources in each of the three energy bands coincide well with the microwave emitting regions (Fig. 4j), and for events \# 26 and 27 the flaring sources in both spectral domains appear elliptical or loop-like. For the 03:59 event (\#27) the HXR source seems to lie over a southern source - the northern source is probably a sunspot associated gyro-resonance source.

\subsection{May 06 microevents}

On May 6, three microflares (\#28-30) were observed: 02:03, 02:26, and 02:38 UT. As shown in Fig. 4k, the 02:26 and 02:38 events have co-located microwave and HXR sources. The 02:03 event occurred when NoRH was not operating, so only the post-event $17-\mathrm{GHz}$ image is shown, and no background-subtraction image could be made. The events \#29 and 30 appear to be harder in HXR than event \#28, as evidenced by the appearance of $12-25 \mathrm{keV}$ sources for \#29 and 30 and none for \#28. 


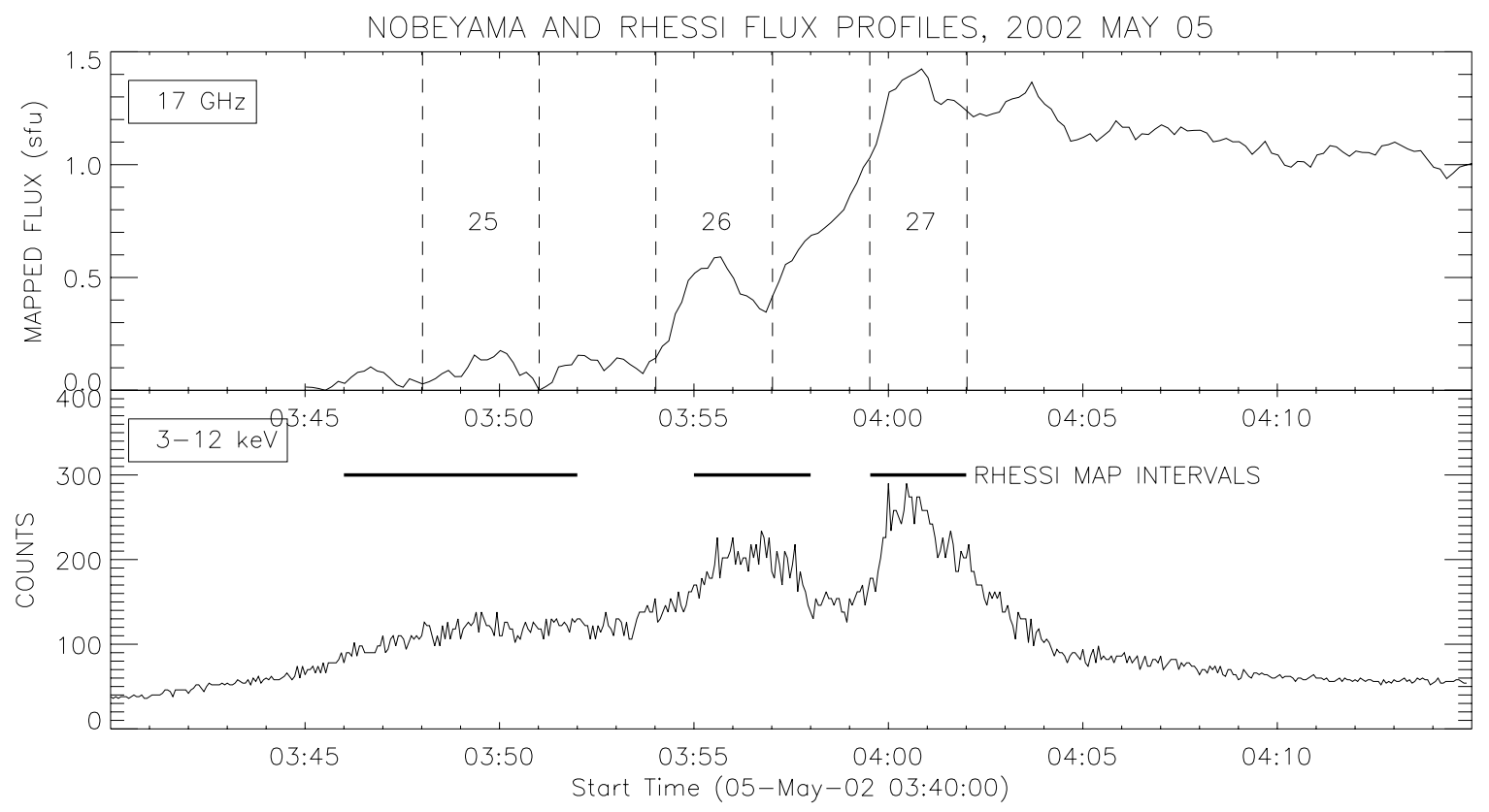

Fig. 3h. 17-GHz NoRH and RHESSI 3-6 keV light curves for events \#25-27. The noise level in the $17 \mathrm{GHz}$ profiles is $\sim 0.014 \mathrm{~K}$.

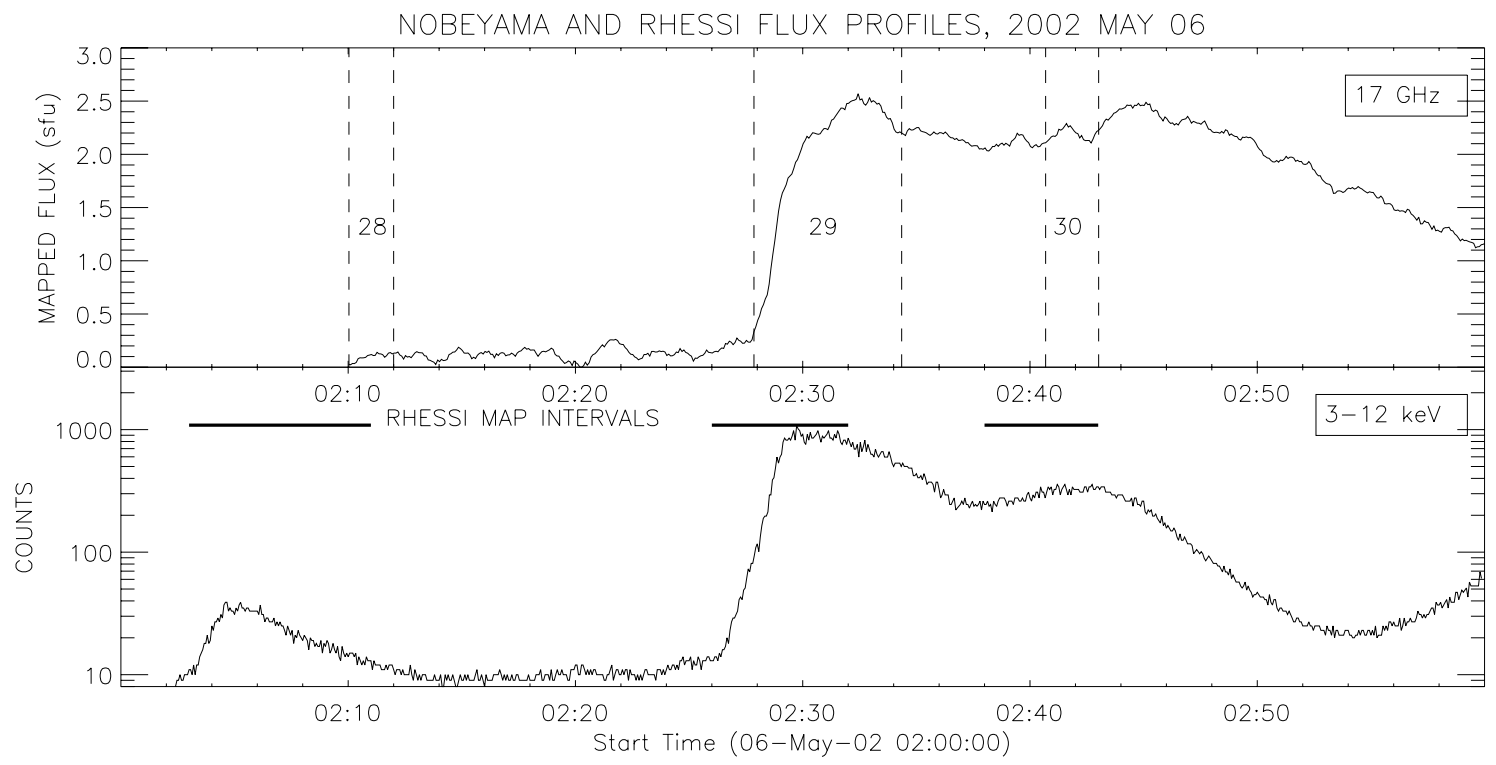

Fig. 3i. 17-GHz NoRH and RHESSI 3-6 keV light curves for events $28-30$. Note that that there was a gap in $17-\mathrm{GHz}$ observations until 02:10 UT, immediately following the peak of HXR event \#28, so only event-decay microwave data were obtained. The HXR data, plotted on a log scale, show that the event lasted until $\sim 02: 12 \mathrm{UT}$. The noise level in the $17 \mathrm{GHz}$ profiles is $\sim 0.013 \mathrm{~K}$.

\section{Nonthermal and thermal components in microflares}

From the RHESSI HXR and microwave polarization measurements, we look for independent evidence of nonthermal components in our sample of microflares. We seek to determine if these nonthermal components appear to be inter-related in their timing, as in normal flares.

\subsection{RHESSI hard X-ray spectra}

Using the OSPEX HXR spectroscopy software (Schwartz et al. 2002), for each of four events selected for this study (\#8, 11, 13, and 26) we have obtained hard X-ray spectra to derive physical properties of the sources. In each case, the spectrum was fitted by one to more components - isothermal continuum plus lines and nonthermal broken powerlaw. The temperatures of the isothermal components are typically $10-20 \mathrm{MK}$; the emission measures range from $10^{45}$ to $10^{47} \mathrm{~cm}^{-3}$, and the indices of the power laws (where determinate) range from 3 to 8 . It is interesting to compare these results with those of Benz \& Grigis (2002).

The spectral data were obtained in time bins averaging 32 , 18,20 , and $30 \mathrm{~s}$ (respectively) for these events. (Due to peculiarities in the software, 18 and 30-s bins result from uneven subdivision of bins between 16 and 20-s and 28 and 32-s.) 


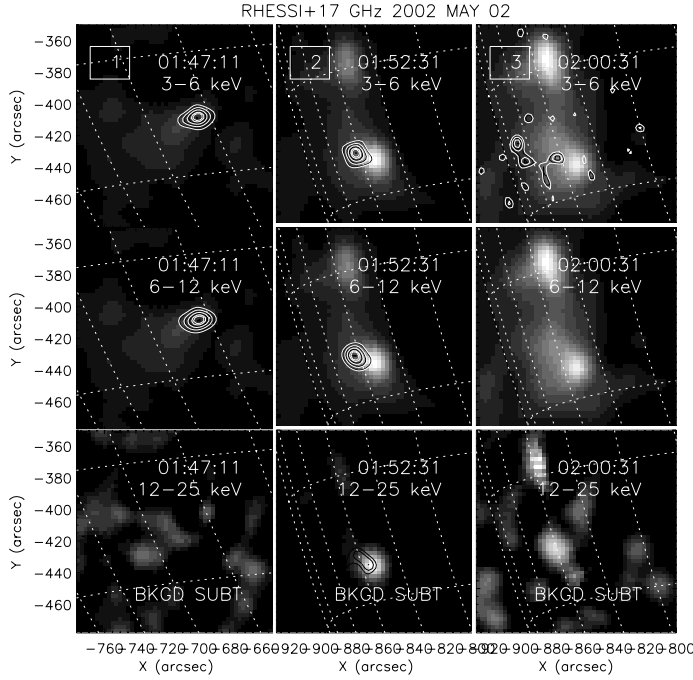

Fig. 4a. Events 1-3. May 2 RHESSI microflare images (contours) superposed on the $17-\mathrm{GHz}$ images. The top and middle rows show $3-6 \mathrm{keV}$ and $6-12 \mathrm{keV}$ HXR contours, respectively, on the $17-\mathrm{GHz}$ image. The bottom row shows $12-25 \mathrm{keV}$ HXR band on the background-subtracted 17-GHz image $\left(\Delta T_{\mathrm{b}}\right)$. The maxima of $T_{\mathrm{b}}$ in the $17 \mathrm{GHz}$ maps are $0.95,1.8$ and $0.90 \times 10^{5} \mathrm{~K}$, for events 1,2 and 3, respectively. In the background-subtracted maps $\Delta T_{\mathrm{b}}$ ranges from -0.97 to $1.2,-1.1$ to 1.0 , and -1.0 to $1.5 \times 10^{5} \mathrm{~K}$, respectively. The background is uncertain due to fluctuations in the bright sunspot-associated source.

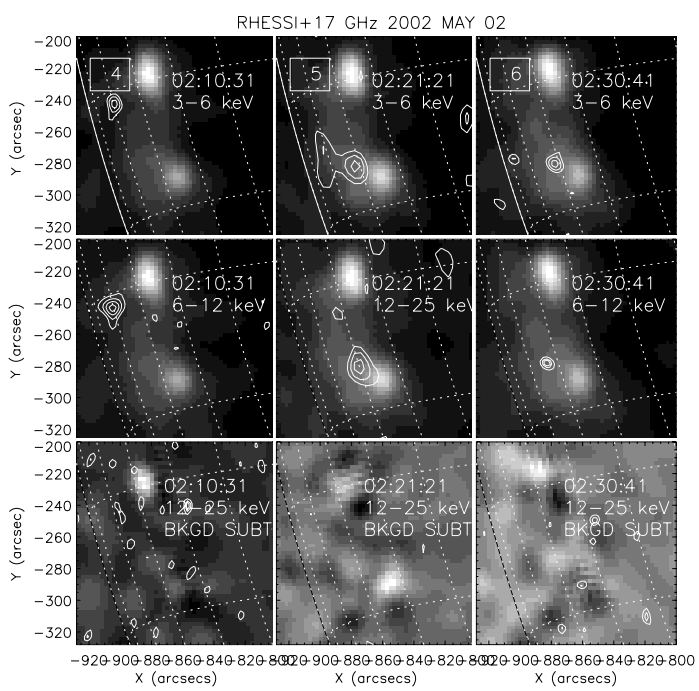

Fig. 4b. Events 4-6. May 2 RHESSI microflare images (contours) superposed on the $17-\mathrm{GHz}$ images. The top and middle rows show 3-6 keV and 6-12 keV HXR contours, respectively, on the $17-\mathrm{GHz}$ image. The bottom row shows $12-25 \mathrm{keV}$ HXR band on the background-subtracted $17-\mathrm{GHz}$ image. The maxima of $T_{\mathrm{b}}$ in the $17 \mathrm{GHz}$ maps are $1.3,1.0$, and $0.96 \times 10^{5} \mathrm{~K}$ in events 4,5 and 6 , respectively. In the background-subtracted maps $\Delta T_{\mathrm{b}}$ ranges from -1.3 to $4.8,-1.5$ to 2.0 and -1.8 to $1.6 \times 10^{4} \mathrm{~K}$, respectively.

These time profiles and time bins are shown in Fig. 5 in 4 plots, each showing $\log$ (flux) in energy bands $3-6,6-12,12-25$, and $25-50 \mathrm{keV}$, with vertical displacements chosen to prevent overlap of the profiles. The dashed curves below the time profiles are the background models subtracted from the data before

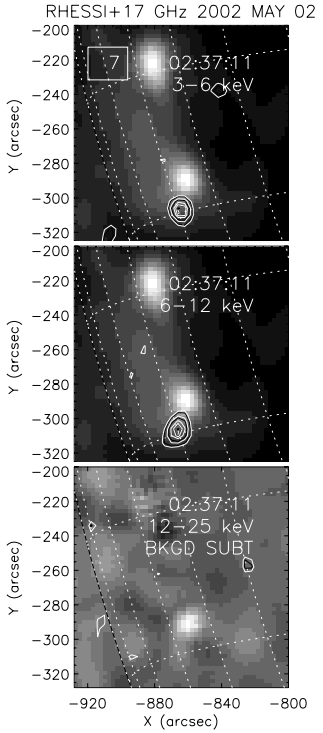

Fig. 4c. Same as previous figure, for event \#7 on May 2. The $T_{\mathrm{b}}$ maximum is $1.0 \times 10^{5}$ and in the background-subtracted map $\Delta T_{\mathrm{b}}$ ranges from -2.2 to $3.3 \times 10^{4} \mathrm{~K}$.

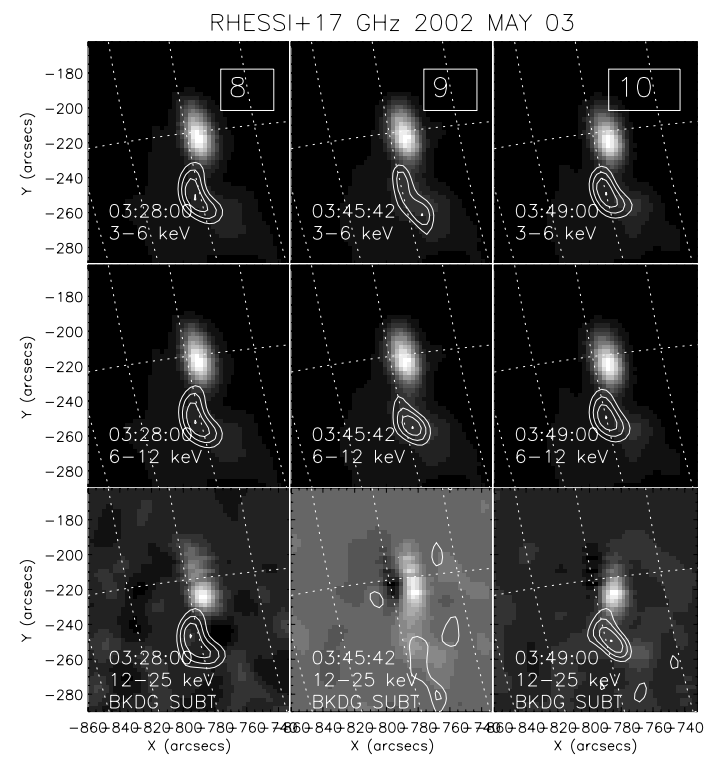

Fig. 4d. Same as previous figure, for events \#8-10 on May 3. The $T_{\mathrm{b}}$ maxima in each event are 4.3, 4.1, and $5.0 \times 10^{5} \mathrm{~K}$, respectively. In the background-subtracted maps $\Delta T_{\mathrm{b}}$ ranges from -0.1 to $0.8,-0.7$ to 1.1 , and -0.4 to $2.1 \times 10^{5} \mathrm{~K}$ respectively.

fitting was done. In some cases there may be post-flare plasma from earlier events that is not accounted for in our background profiles, but corrections for such plasma are beyond the scope of this paper.

In each case, after background subtraction, three models were used to fit the data: (A) isothermal + line emission; (B) isothermal + line emission + broken powerlaw; and $(\mathrm{C})$ double isothermal + line emission. Figure 6 shows the data and spectral fits of the three models near the time of highest inferred 20-keV flux of the powerlaw component (model B) found in each event. 


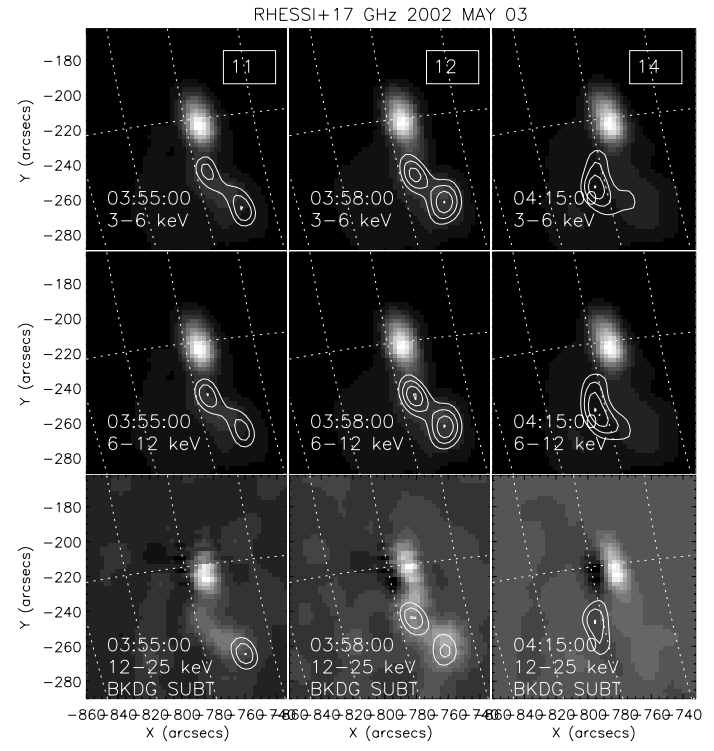

Fig. 4e. Same as previous figure, for events \#11, 12 and 14 on May 3. Event \#13 is skipped over here because it is in a different region. The $T_{\mathrm{b}}$ maxima in each event are $5.0,4.7$, and $4.7 \times 10^{5} \mathrm{~K}$, respectively. In the background-subtracted maps $\Delta T_{\mathrm{b}}$ ranges from -0.3 to $1.5,-0.5$ to 1.4 , and -1.1 to $2.3 \times 10^{5} \mathrm{~K}$ respectively.

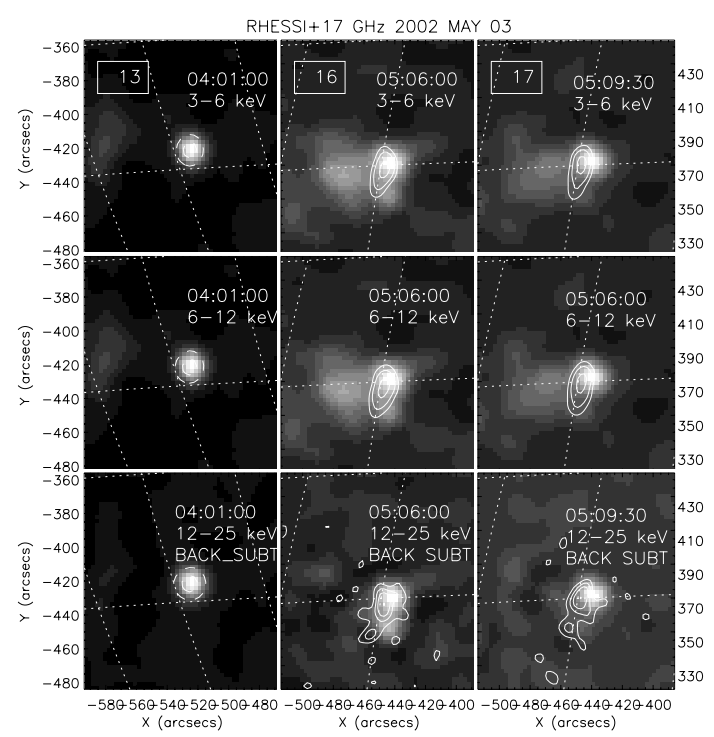

Fig. 4f. Same as previous figure, for events \#13, 16 and 17 on May 3. The $T_{\mathrm{b}}$ maxima in each event are $1.2,0.4$, and $0.6 \times 10^{5} \mathrm{~K}$, respectively. In the background-subtracted maps $\Delta T_{\mathrm{b}}$ ranges from -0.1 to 11.4 , -0.1 to 2.6 , and -1.3 to $4.6 \times 10^{4} \mathrm{~K}$ respectively.

Figure 6 is a $3 \times 4$ matrix of HXR spectra, each of the 4 events in one column, and the 3 models in rows. The normalized residuals ( whose RMS values $=\chi^{2}$ ) are shown at the bottom of each of the plots. For events 8, 11, 13, 26 (Fig. 5), we use the $3 \mathrm{rd}$, 4th, 4 th and 1 st intervals (respectively) to show these spectra.

Using the same times as for Fig. 6, Figs. 7 and 8 show the time history of the parameter fits for models $\mathrm{B}$ and $\mathrm{C}$ for each event. We do not show model A parameter fits, because they can be ruled out in each of the 4 events. For all the events, the normalized residuals (Fig. 6) for model A (first row) are much

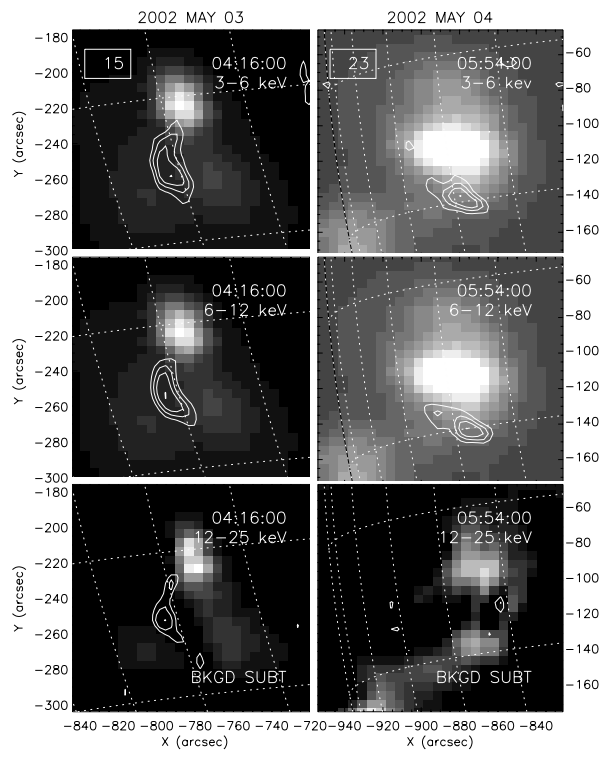

Fig. 4g. Same as previous figures, for events \#15 (May 3) and 23 (May 4). The $T_{\mathrm{b}}$ maxima in each event are 3.8 and $2.8 \times 10^{5} \mathrm{~K}$, respectively. In the background-subtracted maps $\Delta T_{\mathrm{b}}$ ranges from 0 to 1.4 , and -3.6 to $2.8 \times 10^{5} \mathrm{~K}$ respectively. The background of event \#23 is uncertain due to fluctuations in the bright sunspot-associated source.

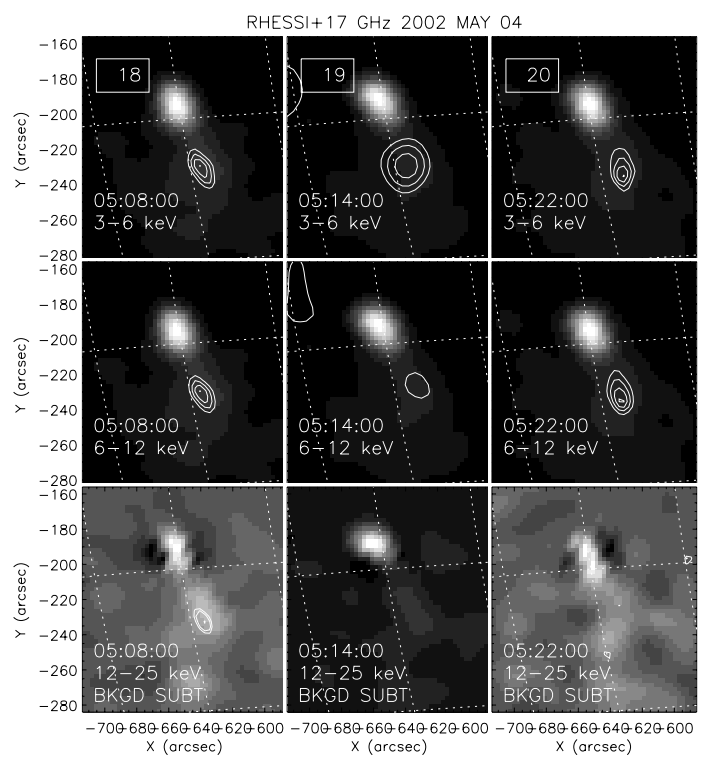

Fig. 4h. Same as previous figures, for events \#18-20 on May 4. The $T_{\mathrm{b}}$ maxima in each event are $3.9,4.8$, and $3.8 \times 10^{5} \mathrm{~K}$, respectively. In the background-subtracted maps $\Delta T_{\mathrm{b}}$ ranges from -3.6 to $6.8,-2.6$ to 2.2 , and -3.2 to $5.6 \times 10^{4} \mathrm{~K}$ respectively.

larger than the normalized residuals for model B and C (2nd and 3rd rows).

We compare models $\mathrm{B}$ and $\mathrm{C}$ for the four events, in an attempt to rule out one of the two models. For event \#8 (Col. 1, Fig. 6), $\chi^{2}$ for model $\mathrm{C}$ is less than $\chi^{2}$ for model $\mathrm{B}$ for the middle 3 timebins, but not the others. However, the jump at the beginning may indicate an impulsive rise of the hard component, easier to explain in a nonthermal model. The residuals (Fig. 6, 2nd and 3rd rows) are so similar for models B and $\mathrm{C}$ they cannot be used to distinguish which model fit is 


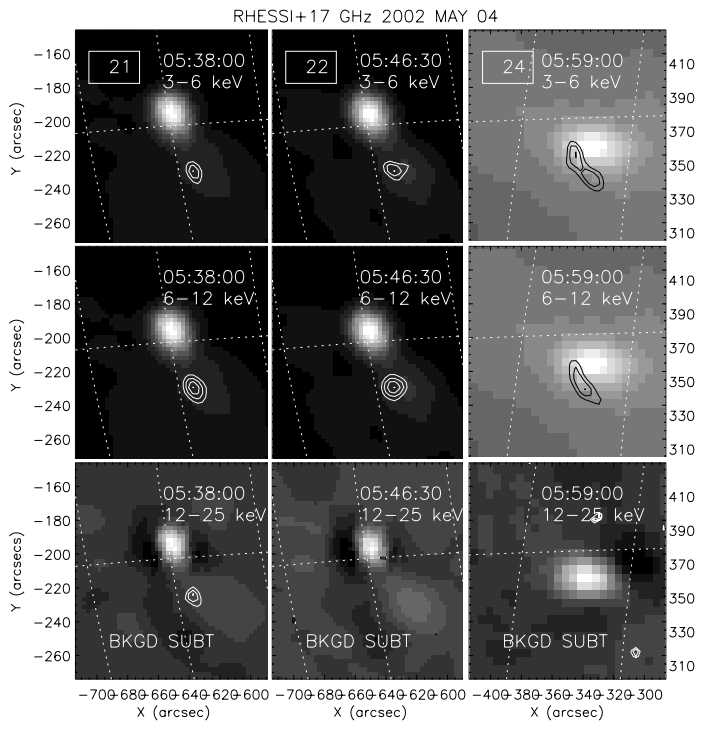

Fig. 4i. Same as previous figures, for events \#21, 22, 24 on May 4. The $T_{\mathrm{b}}$ maxima in each event are 3.9, 3.6, and $0.29 \times 10^{5} \mathrm{~K}$, respectively. In the background-subtracted maps $\Delta T_{\mathrm{b}}$ ranges from -0.5 to $1.3,-0.0$ to 0.29 , and -0.03 to $0.12 \times 10^{5} \mathrm{~K}$ respectively.

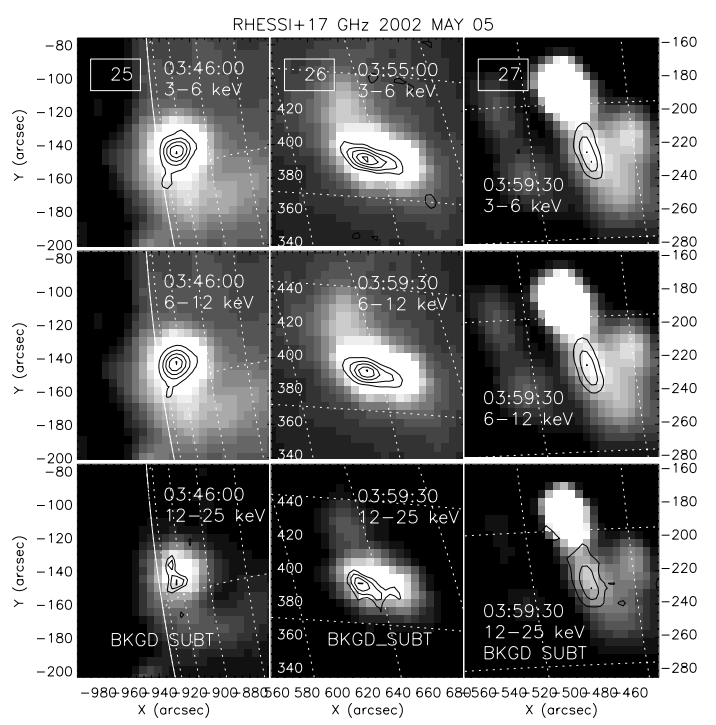

Fig. 4j. Same as previous figures, for events \#25-27 on May 5. The $T_{\mathrm{b}}$ maxima in each event are $2.7,2.9$, and $2.7 \times 10^{5} \mathrm{~K}$, respectively. In the background-subtracted maps $\Delta T_{\mathrm{b}}$ ranges from -0.01 to $1.9,-0.02$ to 2.1 , and -.02 to $1.9 \times 10^{5} \mathrm{~K}$ respectively.

better. For event \#11 (Col. 2), a similar statement can be made for the residuals, except that model B has slightly smaller $\chi^{2}$ for most of the time intervals. The hot component of model $\mathrm{C}$ seems non-physically high, arguing therefore for model B. For event \#13 (Col. 3), model B has about half the $\chi^{2}$ value of model $\mathrm{C}$ at all times. For event \#26, $\chi^{2}$ is smaller for model $\mathrm{B}$ than for $\mathrm{C}$ in 4 out of the 5 time bins. Thus only in events \#13 and 26 can we say with confidence from $\chi^{2}$ that model B fits the data better than model C, although physical arguments may be advanced to favor model $\mathrm{B}$.

The spectral parameters for the model B fits are shown in Fig. 7. Error bars computed for the fits are shown in the plots.

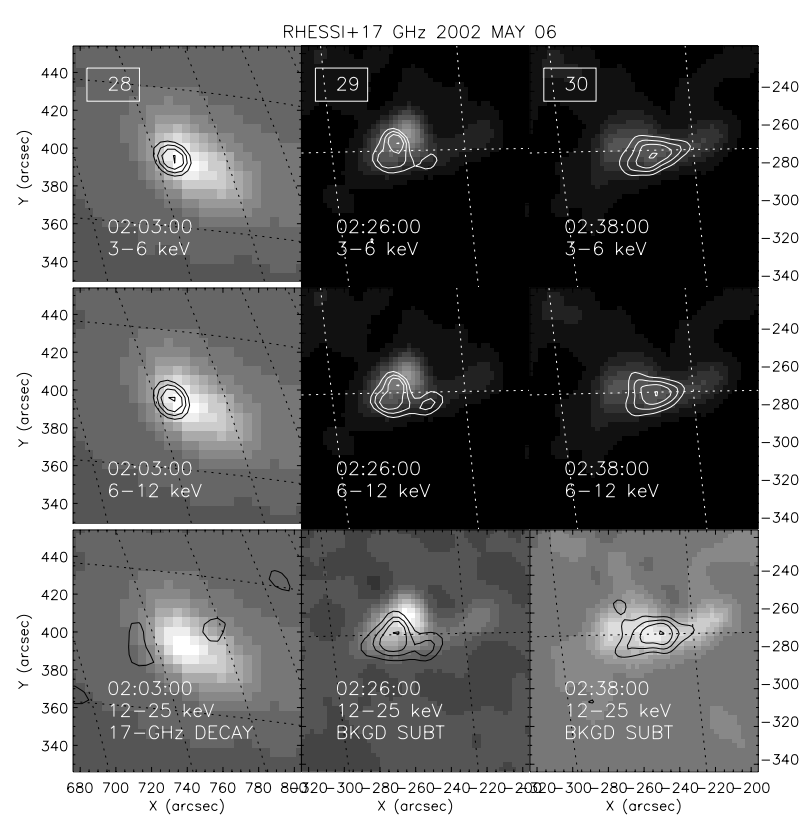

Fig. 4k. Same as previous figures, for events \#28-30. As shown in the time profile Fig. 3i, the microwave data started at 02:10 UT, a few minutes after the peak of HXR in event \#28, so the $17-\mathrm{GHz}$ image (02:10-02:12) is shown for the decay of the event, which lasted until 02:13 in HXR. The $T_{\mathrm{b}}$ maxima in each case were $0.4,2.7$, and $1.4 \times 10^{5} \mathrm{~K}$, respectively. The ranges of the background-subtracted maps were -0.65 to $1.4 \times 10^{5}$ and -8.1 to $8.9 \times 10^{4}$ for events 29 and 30 respectively.

These incorporate an estimated systematic error of $2 \%$ in the $\chi^{2}$ statistic. As shown in the first row, for all events, the emission measure of the isothermal component rises from the start. The temperature (2nd row) for events \#8, 11 and 26 (1st, 2nd and 4 th columns) falls monotonically. This temperature behavior is not seen in event \#13, in which the temperature appears to rise and then fall.

The low energy break of the power law (Fig. 7, row 3) varies between 8 and $16 \mathrm{keV}$ for events \#8 and 11 , but is remarkably constant $(\sim 12 \mathrm{keV})$ for the other two events.

In all 4 events (Fig. 7, row 4), except for isolated time bins, the powerlaw component softens. In events \#8, 11 and 13 the nonthermal flux (5th row) rises at the beginning of the event, but in event \#26 the nonthermal flux starts falling from the first time bin, suggesting that the binning was too coarse to catch the rise. Notably, none of the events shows a clear soft-hard-soft time profile. The $F_{20}$ flux (5th row) seems to peak during the rise of emission measure for events \#8, 11, and 13), consistent with the normal Neupert effect.

Inspecting Fig. 8 for model C, we see some correlated behavior in the time profiles of the cool and and hot plasmas. The emission measure for the cool and hot components (rows 1 and 3) behave rather similarly as functions of time. The same is true for the temperatures (rows 2 and 4), except for the first time bin of event \#8. Thus, to the extent that model $\mathrm{C}$ is preferred, this would imply that the hot and cool component plasmas are both parts of the same distribution.

In summary, the RHESSI observations show clear evidence for nonthermal emission in two of the selected microflares, and 

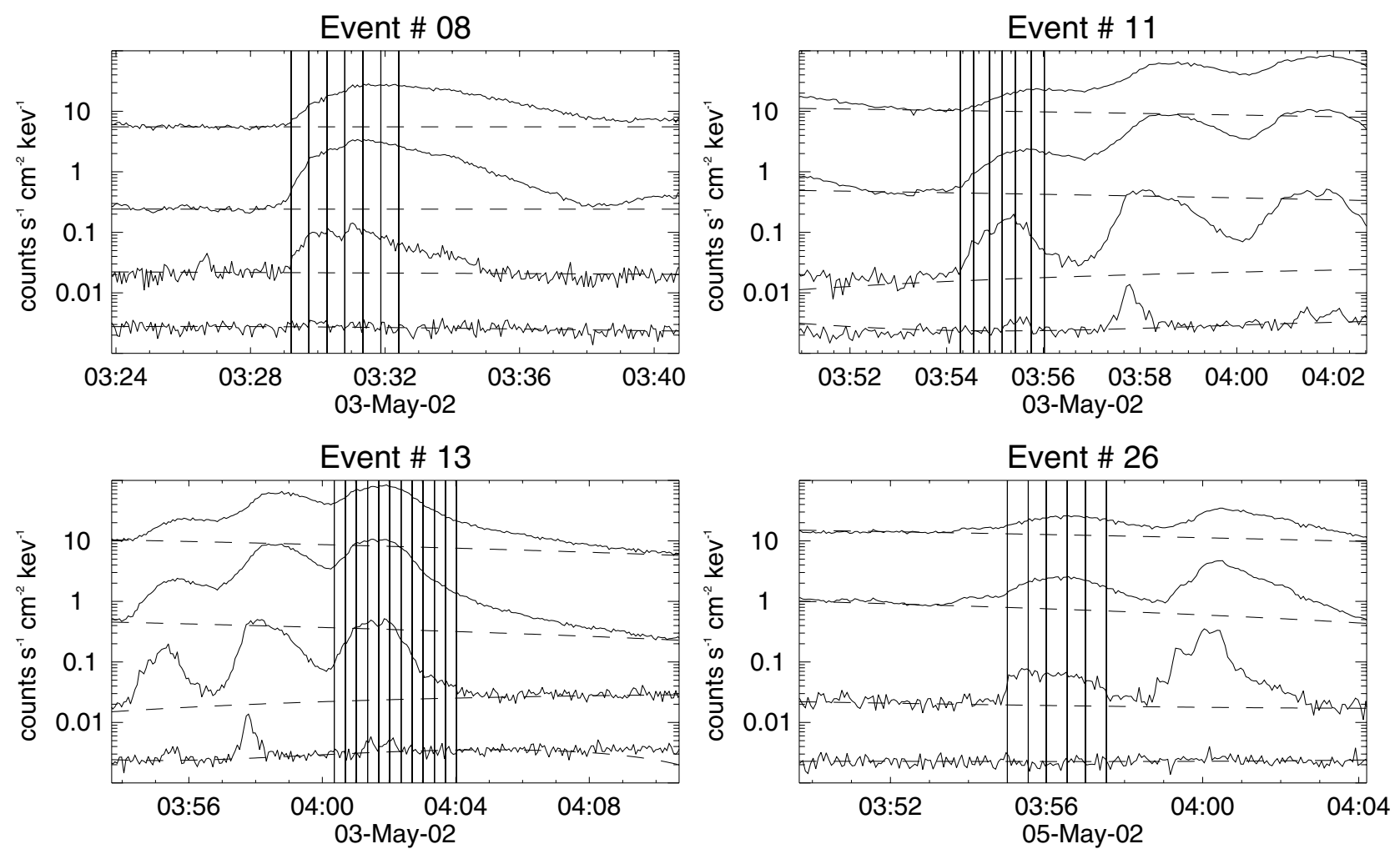

Fig. 5. Four selected HXR events showing time profiles in the energy bands 3-6, 6-12, 12-25, and 25-50 keV (top, 2nd, 3rd and lowest profile, respectively) and the time bins used for spectroscopy. The dashed curves show the background subtracted from the count profiles before model fitting.

possibly in the others. Photon spectral indices range from $\sim 2$ to $\sim 8$, at the beginning of the events. The inferred temperatures of the isothermal component(s) range from $\sim 10$ to $\sim 20 \mathrm{MK}$. The emission measures at the peaks are of order $\sim 10^{46}-10^{48}$, one to three orders of magnitude smaller than moderate flares.

The events observed here, which are of GOES class around $\mathrm{C} 1$, show a trend of increasing emission measure throughout the event, similar to the behavior reported in Benz \& Grigis (2002). The temperature of the thermal component may decrease during the event or reach a peak in conjunction with the X-ray flux. The events are relatively hot (>10 MK), confirming the earlier RHESSI results, which suggests that the observed relation between soft X-ray flare size and temperature (Battaglia et al. 2005) tends to flatten out at small fluxes. The non-thermal component is quite soft, which seems to be typical for small events.

\subsection{Microwave polarization}

Because the microevents are weak we were unable to make $V$ maps, but the time profiles (Figs. 2 and 9) of $I$ and $V$ for four of the events (\#2, 19, 25 and I26) show clearly that these events were polarized (up to $30 \%$ ). This suggests that nonthermal electrons producing gyrosynchrotron emission were present, and supports the RHESSI evidence of powerlaw spectra in some microflares.

\subsection{Neupert effect in microflares}

It is well known that the high energy X-ray time profile looks very much like the derivative of the soft X-ray profile (Neupert 1968). This similarity is called the Neupert effect, and is interpreted to indicate that the same fast electrons which produce the hard X-rays and impulsive microwaves also heat the plasma responsible for the soft X-ray emission (Dennis \& Zarro 1993; Dennis et al. 2003). It is of interest to see if the Neupert effect also exists in microflares, since this would be significant for identifying their energetics and causal processes.

Figure 10 shows the soft X-ray flux (GOES 1-8 A, top curve) and RHESSI band fluxes (intermediate curves) for several microflares. The bottom curve shows the regularized derivative of the soft X-ray profile. This curve shows a striking similarity in timing, and a rough similarity in impulsiveness, to the $12-25 \mathrm{keV}$ flux profile (dotted curve). This similarity shows that the Neupert effect holds down at least to the level of smaller microflares, i.e., to at least the lower B-class level. This suggests that microflares have the same energetic processes as normal flares, only scaled down in magnitude. Note that Qiu et al. (2004) also found the Neupert effect in several microflares.

\section{A synthesis of HXR and microwave source structure}

It is well known that every solar flare is unique in its properties and has its own distinct source structure. This holds true 


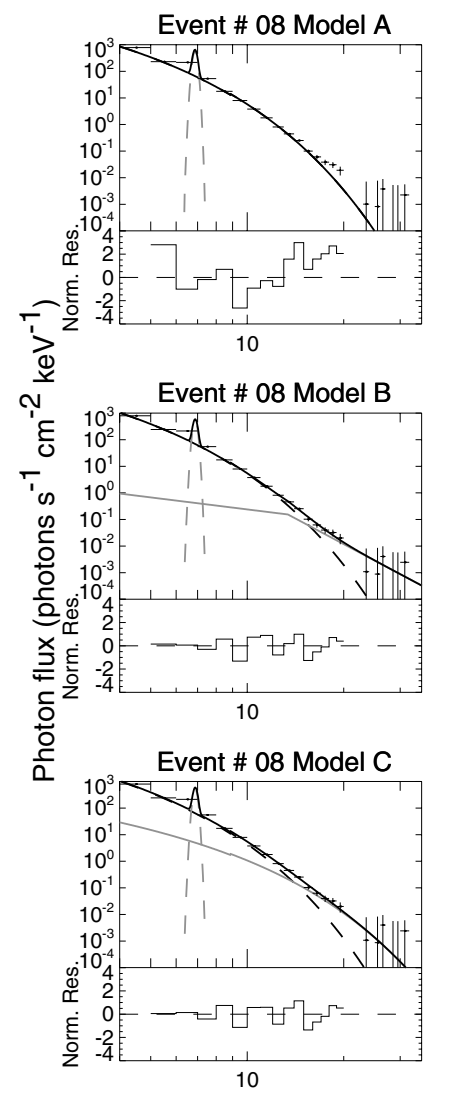

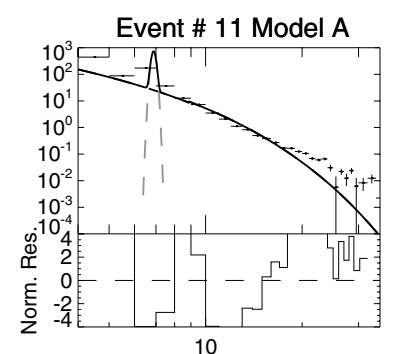

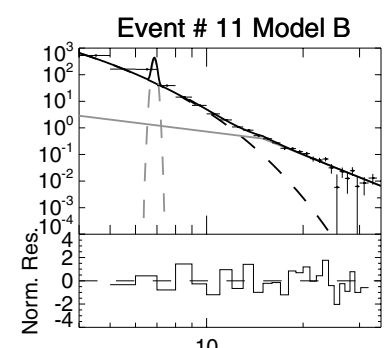

10

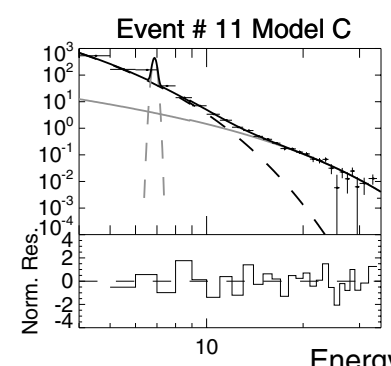

Energ

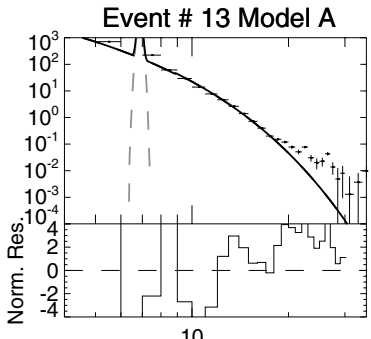

Event \# 13 Model B

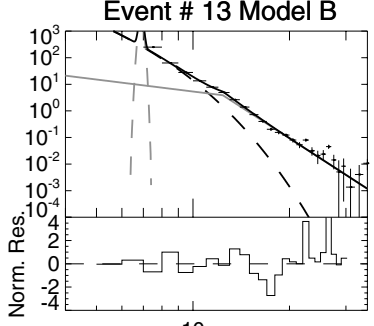

Event \# 13 Model C

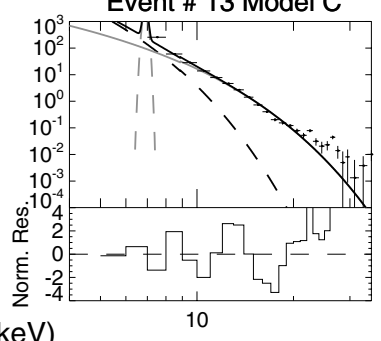

Event \# 26 Model A

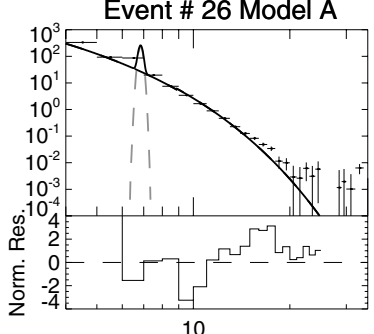

Event \# 26 Model B
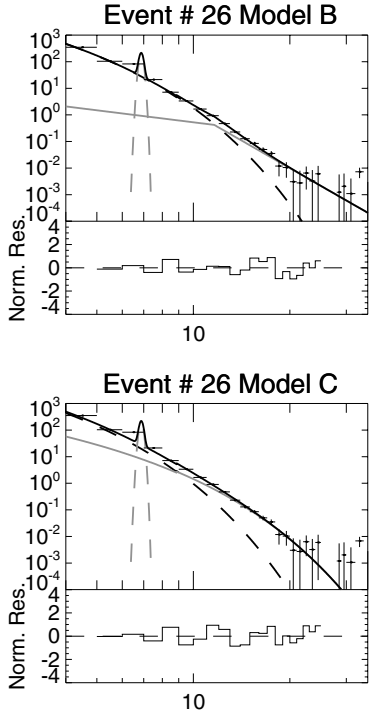

Fig. 6. RHESSI HXR spectral fits to the photon flux (horizontal grey bars and crosses) using 3 models (A, B, C) for the 4 selected events. model A: isothermal + line emission (dashed black curve); model B: isothermal (dashed black curve) + line emission emission (dashed grey curve) + broken powerlaw (solid grey curve); and model C: hotter isothermal (solid gray curve) + line emission (dashed black curve) + cooler isothermal (dashed black curve). In all cases, the continuous black represents the sum of the components. The normalized residuals incorporate a value of $2 \%$ for systematic errors.

also for microflares. Even so there seems to be some pattern in the microflare source structure in hard X-rays relative to that in microwaves. We shall attempt to make some generalization of this relationship of source structure in the two spectral domains. This generalization is based upon the images and locations of 29-30 microbursts observed at $17 \mathrm{GHz}(\mathrm{NoRH})$ and in HXR in the 3-25 keV range (RHESSI). A few examples are shown in Fig. 11.

The most infrequent kind of microbursts observed are those which have exact location and source structure in both HXR and $17 \mathrm{GHz}$. This kind of source must imply very compact flaring loop in both spectral domains-obviously unresolved by the resolution of the present instruments (example: burst \#13, 2002 May 3, 04:01). The most commonly observed structure is the type whose HXR source is nearly coincident with the $17 \mathrm{GHz}$ source, with slight offset of HXR from the $17 \mathrm{GHz}$ source (example: burst \#2, 2002 May 2, 01:52). This particular relative locations and structures have many variations, with the offset in position being quite large (up to several arc seconds) (example: burst \#8, 2002 May 3, 03:28). In such a situation the RHESSI HXR source has a loop structure (must be a filled loop) and most likely is located above the low lying microwave source. Less common than this, but more common than the first category, is that both HXR and $17 \mathrm{GHz}$ sources are elongated and they are coincident with each other - all the way up to 12-25 keV range. (Example: \#26, 2002 May 5 03:55) This structure may simply imply that we are dealing with filled loops in both spectral domains - even at higher energies $12-25 \mathrm{keV}$ there are no obvious foot points. Finally we often come across situations in which the HXR source is at one end and the $17 \mathrm{GHz}$ source is at the other end of a sometimes visible, but usually invisible, loop in between (example: burst \#11, 2002 May 3, 03:55). This is similar to what one often observes in normal flares, when the loop has an asymmetric magnetic field; microwaves appear at the higher field end, and HXR at the lower field end of the flare loop, which allows more precipitation of electrons.

\section{Discussion and conclusions}

It is important to study the radio counterparts of these lowenergy RHESSI microflares, because the radio observations provide a good diagnostic of nonthermal energetic electrons responsible for these microflare events. The availability of imaging observations of solar flares by RHESSI in hard X-rays and gamma rays with arc second resolution, offers us the opportunity to compare the emissions from electrons in similar energy range at two very different wavelength regimes: hard X-rays (bremsstrahlung) and radio (gyrosynchrotron). Both of these 

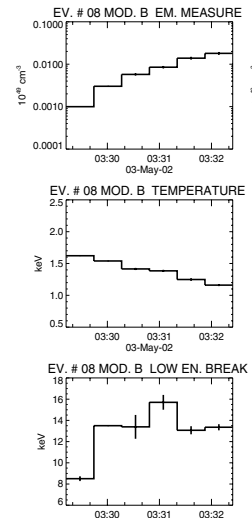

EV.\# 08 MOD. B SP. INDEX
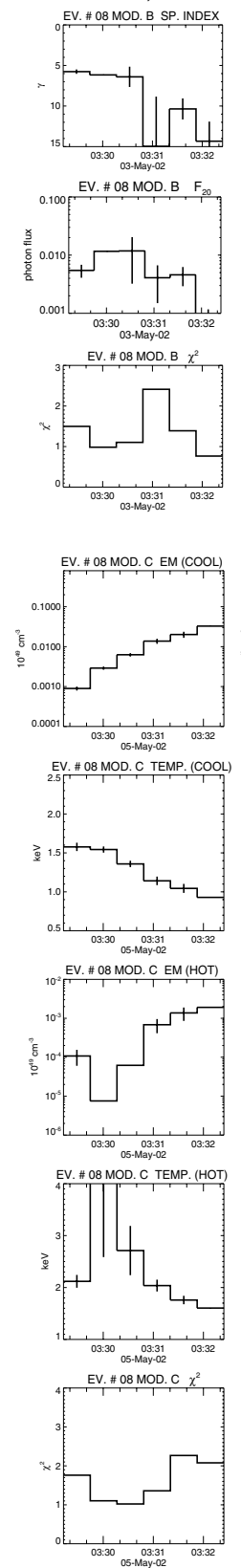
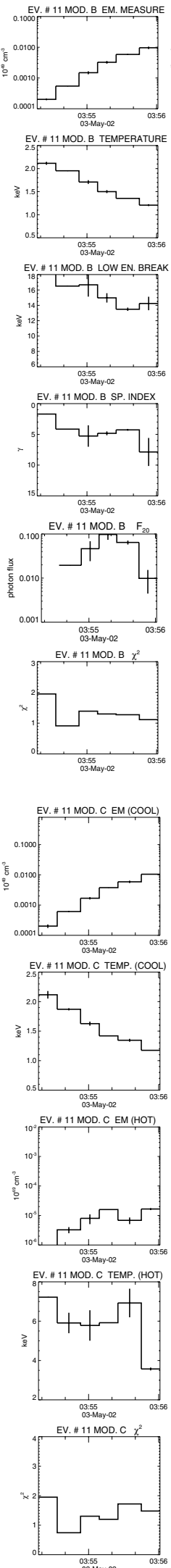
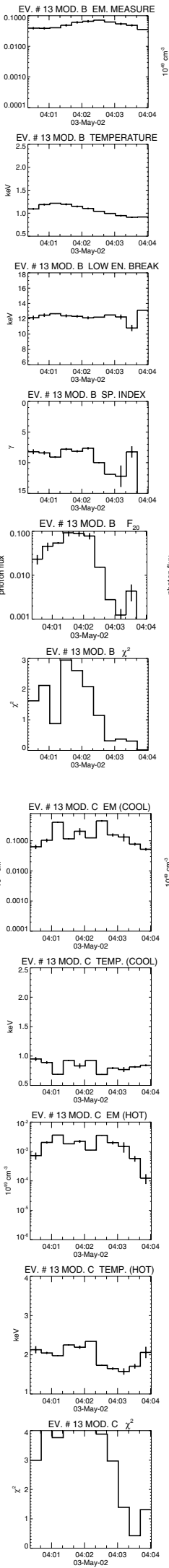
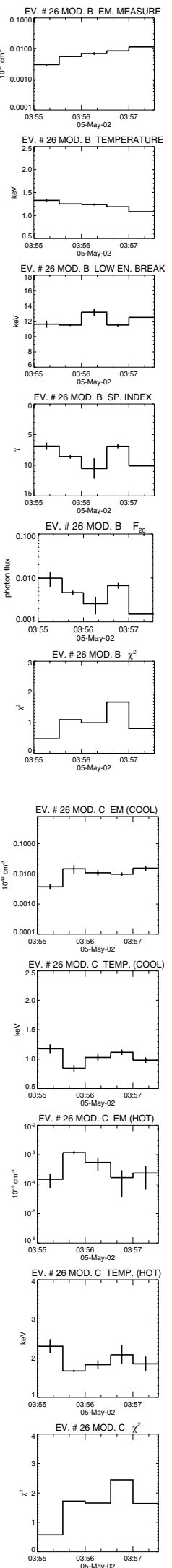

Fig. 7. Time profiles of spectral parameters found for model B (isothermal + powerlaw) for events \#8, 11, 13 and 26. The errorbars have been computed using a value of $2 \%$ for systematic errors.

Fig. 8. Time profiles of spectral parameters found from Model C (double isothermal + powerlaw) for events \#8, 11, 13 and 26. Again the errorbars have been computed using a value of $2 \%$ for systematic errors.

emissions are produced by nonthermal electrons with energies in excess of $100 \mathrm{keV}$, yet the emission mechanisms have very different properties and offer information on different aspects of conditions in the corona. Bremsstrahlung is produced by collisions and therefore requires high densities: in most cases hard X-rays above $100 \mathrm{keV}$ arise from nonthermal electrons striking the chromosphere and images show the footpoints of the coronal loops carrying the accelerated electrons. 


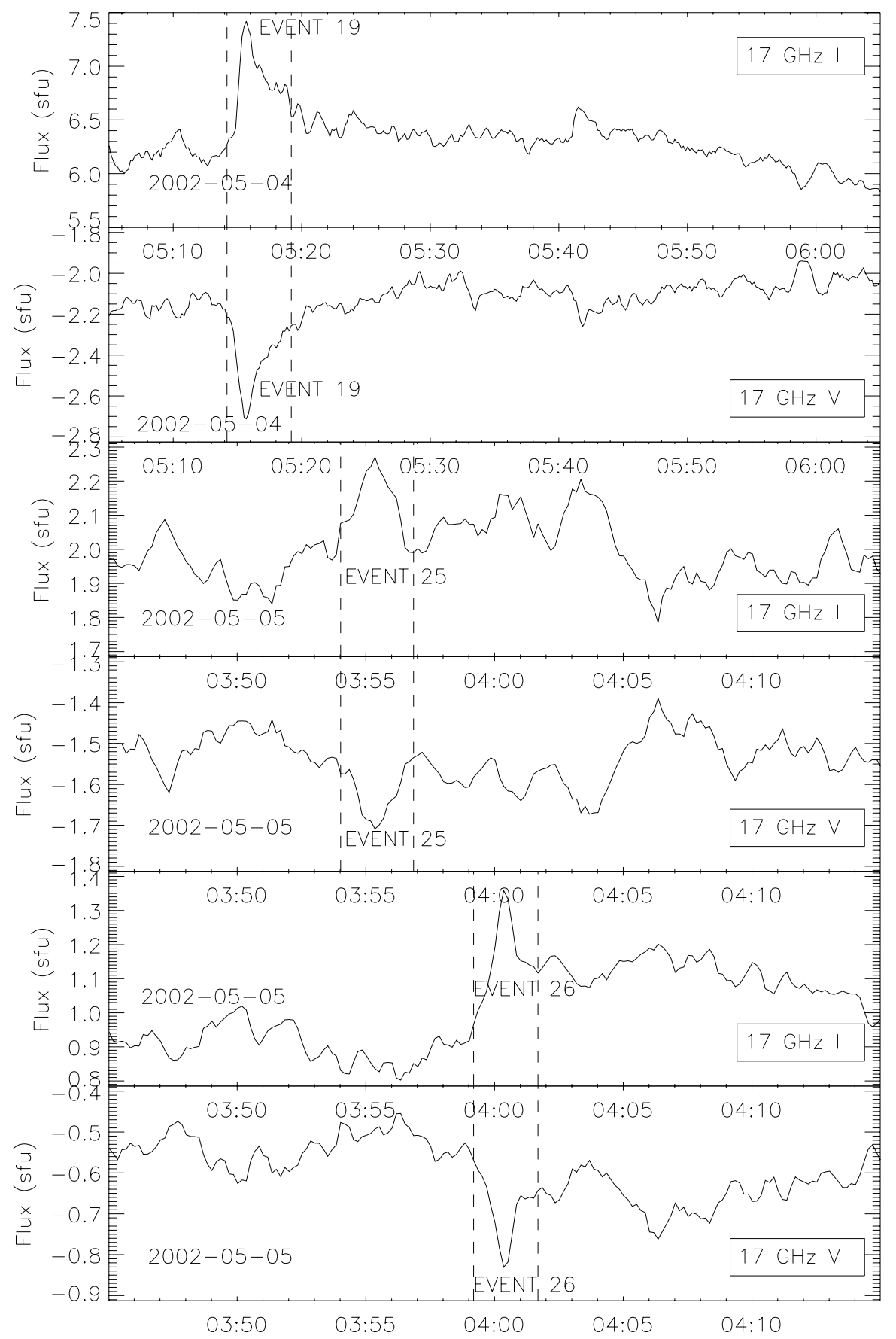

Fig. 9. Time profiles in $I$ and $V$ for three of the events (\#19, 25 and 26) showing that these events were polarized.

Gyrosynchrotron emission is produced when electrons are accelerated by their gyromotion in coronal magnetic fields. This mechanism is extremely efficient and allows us to detect electrons with energies of hundreds of keV even in very small flares. On the other hand, flares in which X-rays above $100 \mathrm{keV}$ (deriving from electrons with energies $\sim 200 \mathrm{keV}$ ) can be imaged are rare because the steeply falling power law spectra do not yield sufficient photons at high energies for image formation.

As mentioned earlier, Krucker et al. (2002) and Benz \& Grigis (2002) have studied RHESSI microflares in the energy range 3-50 keV. Earlier, Lin et al. (2001) investigated
HXR microflares at lower energies using the BATSE SPEC detectors on the Compton Gamma-Ray Observatory whose thresholds were occasionally set as low as $8 \mathrm{keV}$. They found that many nonthermal events are detected above $8 \mathrm{keV}$, but few are seen above $\sim 25 \mathrm{keV}$. Additionally, the generally steep HXR spectra (power-law fits with exponent of 3-7) reveal that most of the flare energy is in the nonthermal electrons at lowest energy. The similarity of frequency vs. energy release distribution of the $>8 \mathrm{keV}$ bursts to that of ARTBs seen in soft X-rays (Shimizu 1992) suggests that these accelerated electrons may provide the energy for ARTBs. 


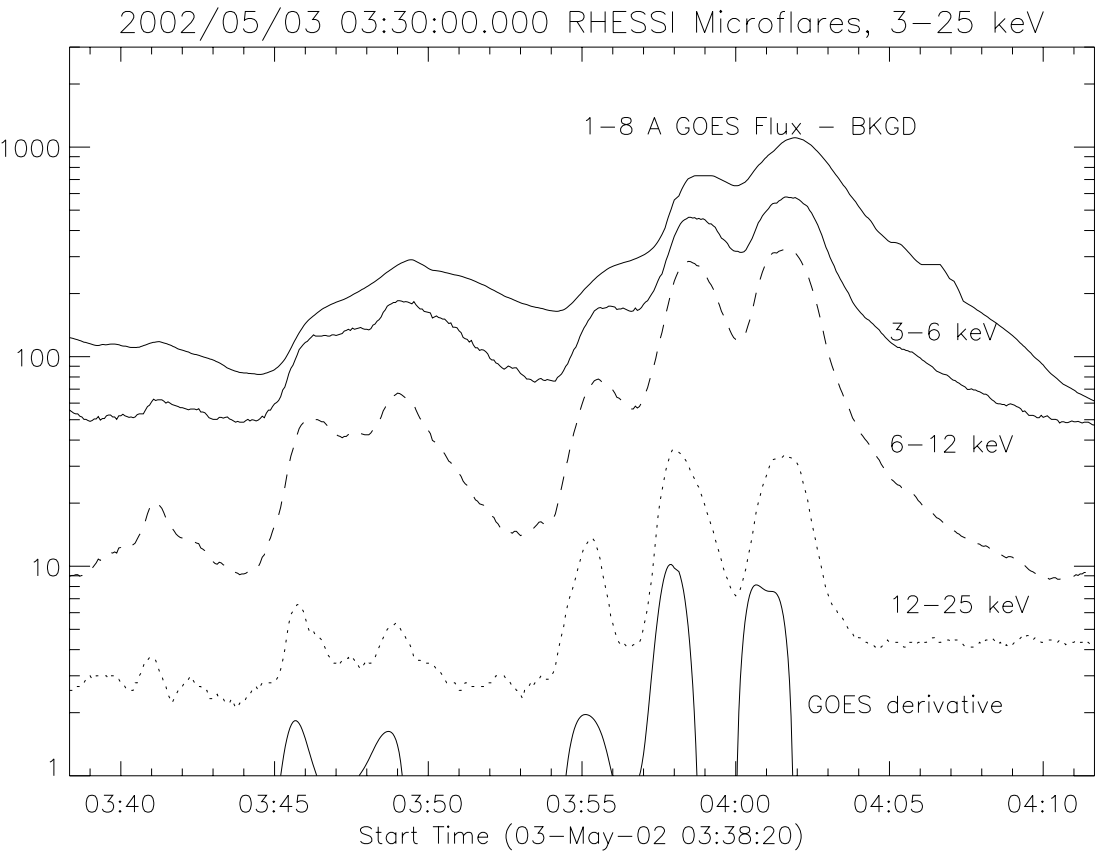

Fig. 10. Soft X-rayy flux (GOES $1-8 \AA$, top curve) and derivatives compared with the HXR profiles for the May 3 events. The similarity of the derivatives and the HXR profiles illustrate the Neupert effect.
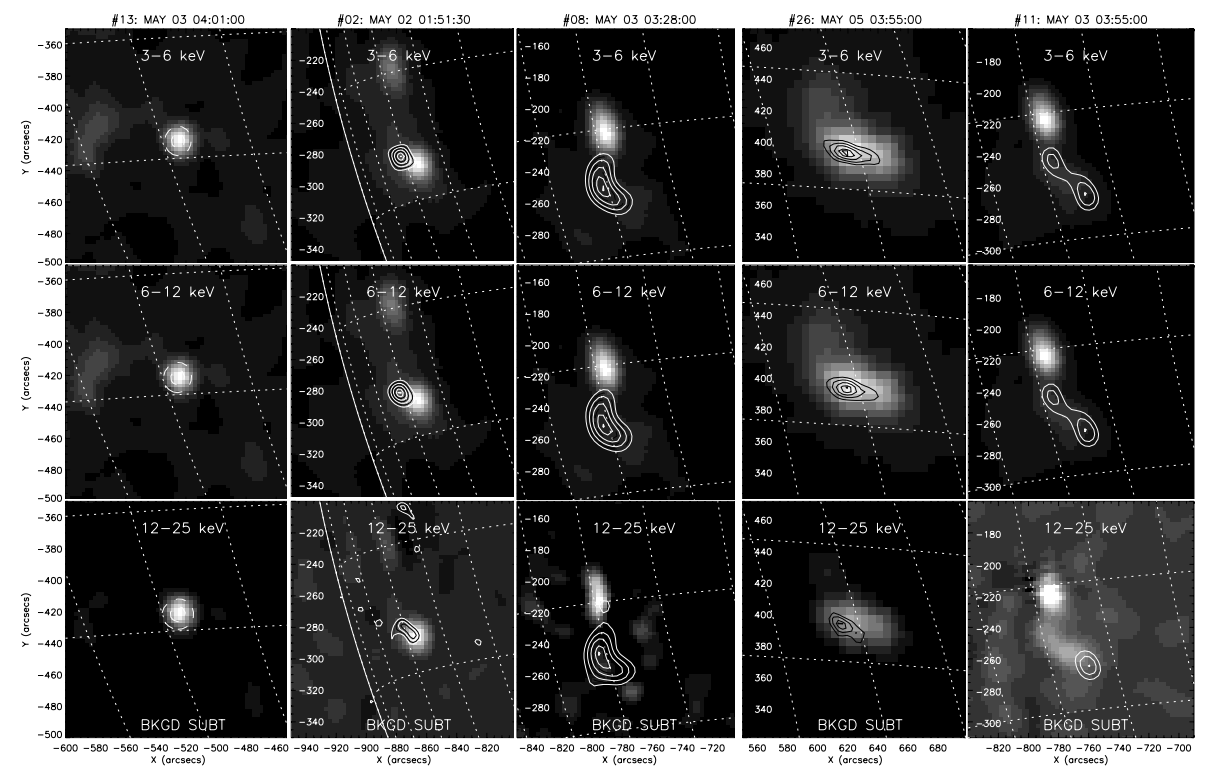

Fig. 11. A sample of 5 microbursts showing the variable morphology of microflare source structure in the HXR and microwave domains. See text for discussion.
Qiu et al. (2004) made a comprehensive study of $760 \mathrm{mi}-$ croflares of which 200 overlapped in time with the Owens Valley Solar Array (OVSA) operating in the frequency range 1.0-18.0 GHz. The RHESSI microflares selected by Qiu et al. had GOES classification class of B2 or above. About 56 microflares or $40 \%$ of those falling in the OVSA time zone were observed simultaneously in microwaves by OVSA (microwave peak flux 1-10 sfu). The OVSA observations were very useful for providing the microwave spectra of microbursts along with photon energy spectra in hard X-rays, but they had no imaging observations. The emphasis in our paper is on imaging properties of the microbursts in both hard $\mathrm{X}$-ray and microwaves, and their relative positions as well as comparison with MDI magnetograms and EIT observations. Using BBSO $\mathrm{H} \alpha$ data along with RHESSI imaging data Liu et al. (2004) studied 12 microflares (A2-B3 class) in the energy range 3-15 keV. Consistent with our observations they found that most compact microflares were located near magnetic neutral lines, implying that we are dealing with small loops. They also found elongated HXR sources connecting two $\mathrm{H} \alpha$ footpoints located in opposite polarity magnetic fields; this is suggestive of flaring loops being sources of microflares. This is identical to what we have found. Further they also found - as we suggest - that the microflares seem to behave like ordinary normal flares. In fact using TRACE data showing EUV jets being associated with some microflares they suggest that magnetic reconnection may play a role in producing microflares (cf. Kundu et al. 1995).

From spectral studies, Qiu et al. found that the emissions in the spectral domains were nonthermal, especially at peak times; they found that the mean value of the photon spectral index, $\gamma_{\mathrm{x}}$, was $5.3 \pm 0.7$ and the mean value of the radio 
spectral index, $\delta_{\mathrm{r}}$, was $3.8 \pm 1.2$, with a mean difference in spectral index between the two $3.0 \pm 0.6$, assuming that nonthermal hard X-rays were emitted by thick target sources. Consistent with our results they found that nonthermal microwave emission can arise from very small microflares, and that most emission in hard X-rays was limited below $25 \mathrm{keV}$, only rarely did they go up to $30 \mathrm{keV}$. The discrepancy in the spectral index found in HXR and microwaves is higher (3.0) than that found in large or normal flares (1.0). Much work needs to be done in the RHESSI and microwave spectral analysis to resolve the origin of this large discrepancy for microflares and normal flares.

In some microevents correlation between the timing of the peak intensity in HXR and $17 \mathrm{GHz}$ is low; the best example of such a situation is the pair of events \#18 and 19. Also there seems to be some delay (both positive and negative) between HXR and $17 \mathrm{GHz}$ peaks, at least in a significant fraction of the cases.

When we are discussing RHESSI-observed hard X-ray microflares along with their microwave $(17 \mathrm{GHz})$ counterparts, it is appropriate to mention briefly the "active region transient brightenings" (ARTBs) first discovered by Shimizu (1992) from Yohkoh/SXT data. The ARTBs are essentially microflares in soft X-rays, and their microwave counterparts have been studied by Gopalswamy et al. $(1994,1997)$ using the VLA at 2 and $3.6 \mathrm{~cm}$, by White et al. (1995) using the NoRH at $17 \mathrm{GHz}$, and by Gary et al. (1997) using the OVSA at 45 frequencies between 1 and $18 \mathrm{GHz}$. White et al. analyzed four ARTBs at $17 \mathrm{GHz}$ and found that the peak $17 \mathrm{GHz}$ flux was close to that predicted on the basis of thermal bremsstrahlung emission from the X-ray emitting material $\left(T \sim 6-7 \times 10^{6} \mathrm{~K}\right.$, EM $\sim 2-5 \times 10^{47} \mathrm{~cm}^{-3}$ ), and the microwave time profiles were rather gradual; thus they concluded that these microwave microflares were thermal in nature. On the other hand, the studies by Gopalswamy et al. and Gary et al., based upon spectra, fast time structure and in some cases polarization information, concluded that a significant fraction of the microflares in microwaves associated with ARTBs in soft X-rays were nonthermal in nature. In the present case, the RHESSI-related microwave microflares typically have all the characteristics of nonthermal phenomena - sharp peak, polarization, and short duration.

We have detected microwave (17-GHz) counterparts of RHESSI microflares observed in the energy range 3-25 keV. In general, the microwave emission comes from the foot points (for higher energies), and from the entire small (mini) flaring loop (for lower energies). The relative positions of microwaves and hard X-rays in the higher energy channels follow the pattern of behavior in normal flares. Sometimes the two (microwave and hard X-ray) sources coincide, at other times the two are at opposite ends of the mini-flaring loop. One sees the mini flaring loops clearly in NoRH and RHESSI images. The hard X-ray spectrum of a typical microwave-associated RHESSI microflare can be fit by an isothermal component (EM a few $\times 10^{46} \mathrm{~cm}^{-3}$ at 3-6 keV) at low energies and (sometimes) a nonthermal component (with slopes in the range $\sim-4$ to -9 around the peak) at higher energies. The time profiles of different components of the $17-\mathrm{GHz}$ active region show that successive events may come from different component sources. Sometimes one observes compact, unresolved co-located HXR and microwave microevents.

Acknowledgements. The research of M.R.K. and V.I.G. was supported by NASA grants NNG 05GM56G, NAG5-12860, NNG 04GG33G, and NSF grant ATM 0233907. E.J.S. was supported at NASA/GSFC by grants NAG5-10180 and NNG06GB636. The work of PCG is supported by the Swiss National Science Foundation (grant 200020-105366).

\section{References}

Benz, A. O., \& Grigis, P. C. 2002, Sol. Phys., 210, 431

Battaglia, M., Grigis, P. C., \& Benz, A. O. 2005, A\&A, in press

Dennis, B. R., \& Zarro, D. M. 1993, Sol. Phys., 146, 177

Dennis, B. R., Veronig, A., Schwartz, R. A., et al. 2003, Adv. Sp. Res., 32,2459

Gary, D. E., Hartl, M. D., \& Shimizu, T. 1997, ApJ, 477, 958

Gopalswamy, N., Payne, T. E. W., Schmahl, E., et al. 1994, ApJ, 437, 522

Gopalswamy, et al. 1997, ApJ, 491, L115

Krucker, S., Christe, S., Lin, R. P., et al. 2002, Sol. Phys., 210, 445

Kundu, M. R., Raulin, J .P., Nitta, N., et al. 1995, ApJ, 447, L135

Kundu, M. R. 1998, in Solar Physics with Radio Observations, Proc. Nobeyama Symp., 111

Lin, R. P., Schwartz, R. A., Kane, S. R., et al. 1984, ApJ, 283, L421

Lin, R. P., Feffer, P. T., \& Schwartz, R. A. 2001, ApJ, 557, L125

Lin, R. P., Dennis, B. R., Hurford, G. J., et al. 2002, Sol. Phys., 210, 1

Liu, W., Jiang, Y. W., Liu, S., \& Petrosian, V. 2004, ApJ, 604, 442

Neupert, W. M. 1968, ApJ, 153, L59

Qiu, J., Lee, J., \& Gary, D. E. 2004, ApJ, 612, 530

Schwartz, R. A., Csillaghy, A., \& Tolbert, A. K. 2002, Sol. Phys., 210, 165

Shimizu, T., Tsuneta, S., Acton, L. W., et al. 1992, PASJ, 44, L147

Smith, D. M., Lin, R. P., \& Turin, P. 2002, Sol. Phys., 210, 33

Van Beek, H. F., Hoyng, P., Lafleur, B., \& Simnett, G. M. 1980, Sol. Phys., 65, 39

White, S., Kundu, M. R., Shimizu, T., Shibasaki, K., \& Enome 1995, ApJ, 450, 435 